\title{
Efficacia e sicurezza dei nuovi farmaci anticoagulanti orali rispetto al warfarin nella profilassi cardioembolica del paziente con fibrillazione atriale non valvolare. Più luci che ombre
}

Efficacy and safety of new oral anticoagulants compared with warfarin in cardioembolic prophylaxis of patients with non valvular atrial fibrillation. More lights than shadows

\section{Luca Masotti $^{\text {a,* }}$, Mario Di Napoli ${ }^{b}$, Walter Ageno ${ }^{c}$, Davide Imberti ${ }^{d}$, Daniel Godoy ${ }^{e}$, Grazia Panigada ${ }^{f}$, Niccolò Napoli ${ }^{f}$, Giancarlo Landini ${ }^{g}$, Roberto Cappelli ${ }^{h}$, Ido Iori ${ }^{i}$, Domenico Prisco ${ }^{j}$, Giancarlo Agnelli ${ }^{k}$}

\footnotetext{
${ }^{a}$ UO Medicina Interna, Ospedale di Cecina, AUSL 6 Livorno

' UOC Neurologia, Ospedale Generale San Camillo de' Lellis, AUSL Rieti

'Dipartimento di Medicina Clinica, Università dell'Insubria, Varese

¿ UO Medicina Interna, Ospedale di Piacenza

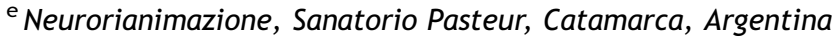

f UO Medicina Interna, Ospedale di Pescia, AUSL 3 Pistoia

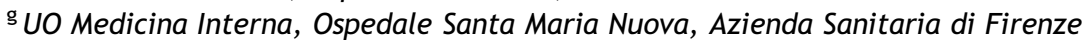

${ }^{\mathrm{h}}$ Centro Trombosi, Università degli Studi di Siena

' UO Medicina Interna, Arcispedale S.ta Maria Nuova, Azienda Ospedaliera di Reggio Emilia

${ }^{j}$ Dipartimento di Area Critica Medico-Chirurgica, Università degli Studi di Firenze

${ }^{\mathrm{k}}$ Medicina Interna e Vascolare-Stroke Unit, Azienda Ospedaliera di Perugia
}

\section{KEYWORDS}

Atrial fibrillation;

Stroke;

Dabigatran;

\begin{abstract}
Summary
Introduction: The prophylaxis of thromboembolic events represents a key point in the modern management of patients with non valvular atrial fibrillation (AF), both paroxysmal and persistent/ permanent. Up to now, vitamin $\mathrm{K}$ antagonist (VKA) drugs are the first choice in thromboembolic
\end{abstract}

\footnotetext{
* Corrispondenza: UO Medicina Interna, Ospedale di Cecina, via Montanara, loc. Ladronaia - 57023 Cecina, Livorno.

E-mail: luca.masotti@tin.it (L. Masotti).
} 
Rivaroxaban;

Apixaban. prophylaxis. Their treatment limitations have lead to development and clinical experimental use of new molecules aimed to overcome their limits. The new oral anticoagulants, such as dabigatran, a direct inhibitor of thrombin or rivaroxaban and apixaban, direct inhibitors of activated factor $\mathrm{X}$, have been compared to warfarin in randomized clinical phase three trials (RCTs) for thromboembolic prevention in patients with non valvular AF with the aim to demonstrate their non inferiority when compared to warfarin. The results of these trials have been recently published. In this article the authors review the results of efficacy and safety of these three more recently published large RCTs.

Conclusions: All RCTS, RE-LY for dabigatran, ROCKET-AF for rivaroxaban and ARISTOTLE for apixaban met the study end-points and demonstrated a good safety profile of each new oral anticoagulant, so promising a new era for thromboembolic prevention therapy in AF.

(c) 2012 Elsevier Srl. All rights reserved.

\section{Introduzione}

La fibrillazione atriale (FA) rappresenta la più diffusa aritmia cardiaca nella popolazione generale e nella pratica clinica ospedaliera [1-3]. Studi di popolazione condotti alla fine degli anni Novanta negli Stati Uniti hanno evidenziato un tasso di prevalenza della FA nella popolazione generale dello $0,95 \%$, con una stima di 2,3 milioni di americani affetti da FA $[4,5]$; in Europa, invece, la prevalenza nella popolazione generale sarebbe addirittura del 5,5\%, come evidenziato nello studio effettuato a Rotterdam [6]. La prevalenza della FA aumenta con l'età: i dati americani indicano che la prevalenza varia dallo $0,1 \%$ nei soggetti di età $<55$ anni al $9 \%$ nei soggetti di età $>80$ anni [4], mentre i dati europei indicano prevalenze variabili dallo $0,7 \%$ nella popolazione della fascia d'età 55-60 anni al 17,8\% nella popolazione di età $>85$ anni $[6,7]$.

Negli ultimi trent'anni l'incidenza della FA è aumentata del $12 \%$ circa [5]; le stime previsionali indicano, inoltre, un ulteriore aumento esponenziale della prevalenza della FA nei prossimi cinquant'anni, con circa il $50 \%$ dei pazienti ultraottantenni affetti [4,5]. Nel 2050 si prevedono negli Stati Uniti, a seconda della stime, tra i 5,6 milioni [4] e i 12,1 milioni di soggetti affetti da FA mantenendo costante l'attuale incidenza, ma il dato potrebbe salire a 16 milioni circa mantenendo costante l'attuale trend di incremento dell'incidenza [5].

La FA rappresenta più di un terzo delle aritmie cardiache riscontrabili nella pratica clinica ospedaliera [1]. In un recente studio osservazionale condotto nei reparti di Medicina Interna italiani (FALP Study) [8] la prevalenza di FA è risultata pari al $18,2 \%$ dei pazienti ricoverati.

La FA aumenta il rischio di tromboembolismo arterioso cerebrale (ictus ischemico cardioembolico) e sistemico $[9,10]$. L'incidenza di ictus ischemico è nell'ordine del $5 \%$ l'anno nei pazienti con FA, con un incremento del rischio di ictus di 2-7 volte rispetto ai soggetti in ritmo sinusale [1-3]. Il rischio incrementa ulteriormente nella popolazione anziana. Sono ad alto rischio di tromboembolismo arterioso i pazienti ultrasettantacinquenni, con FA associata a stenosi mitralica severa, protesi valvolare meccanica, anamnesi positiva per pregresso evento cerebrovascolare embolico $[1-3,11]$. A rischio moderato sono i pazienti di sesso femminile, con ipertensione arteriosa, diabete mellito, scompenso cardiaco congestizio con ridotta frazione di eiezione $(<40 \%)$ [1-3,11]. A basso rischio, ma sempre sostanziale, sono i soggetti di età compresa tra i 65 e i 75 anni, con malattia vascolare (cardiopatia ischemica, arteriopatia obliterante periferica) e quelli con tireotossicosi $[1-3,11]$.

\section{La valutazione del rischio tromboembolico nella fibrillazione atriale}

Il rischio di tromboembolismo arterioso nei pazienti con FA può essere calcolato mediante pratiche scale prognostiche, la più diffusa delle quali è la $\mathrm{CHADS}_{2}$ [12], recentemente modificata nella $\mathrm{CHA}_{2} \mathrm{DS}_{2}$-VASC [1] (Allegato 1) e proposta dalla European Society of Cardiology (ESC) come scala prognostica di riferimento per il calcolo del rischio tromboembolico [1]. Gli ictus ischemici cardioembolici hanno una prognosi peggiore rispetto a quelli aterotrombotici e lacunari $[13,14]$. La lesione ischemica, infatti, è generalmente più estesa e ha un più elevato rischio di trasformazione emorragica. Ne consegue un rischio di mortalità e disabilità in fase acuta, a 30 giorni e a 12 mesi, superiore rispetto agli altri sottotipi ictali [15]. La prevenzione del tromboembolismo arterioso cerebrale e sistemico rappresenta pertanto un punto fondamentale nel moderno approccio alla FA, sia parossistica sia persistente/permanente [1-3].

A oggi, gli antagonisti dalla vitamina K (Vitamin K Antagonist, VKA) hanno dimostrato di essere il presidio farmacologico più efficace nella prevenzione dell'ictus ischemico cardioembolico e del tromboembolismo sistemico, costituendo la prima scelta per la profilassi antitromboembolica nei pazienti con FA [1-3]. I farmaci VKA riducono il rischio relativo di ictus ischemico/embolismo sistemico del $64 \%$ (intervallo di confidenza al 95\%, IC 95\%: 49-74\%) rispetto al placebo e del 39\% (IC 95\%: 19-53\%) rispetto all'acido acetilsalicilico (ASA) [16]. Anche studi più recenti, come il Birmingham Atrial Fibrillation Treatment of the Aged Study (BAFTA) e il Warfarin versus Aspirin for Stroke Prevention in Octagenarians (WASPO) confermano la superiorità dei farmaci VKA nei confronti dell'ASA nella profilassi antitromboembolica dei pazienti anziani con FA $[17,18]$. Il vantaggio nella profilassi antitromboembolica del paziente con FA è comunque realmente evidente solo quando il tempo in cui l'International Normalized Ratio (INR) viene mantenuto nel range terapeutico (Time in Therapeutic Range, TTR) risulta almeno superiore al 40\% [19]. L'ASA è considerato farmaco di seconda scelta nei pazienti con FA ad alto rischio tromboembolico, in quanto riduce il rischio relativo di ictus rispetto al placebo solamente del 19\% (IC 95\%: 1-35\%) [16]. Gli studi Atrial fibrillation Clopidogrel Trial with Irbesartan for prevention 
of Vascular Events-Warfarin (ACTIVE-W) [20] e ACTIVE-A (Atrial fibrillation Clopidogrel Trial with Irbesartan for Prevention of Vascular Events-Aspirin) [21], che hanno confrontato l'associazione di ASA e clopidogrel rispettivamente contro warfarin e ASA da solo, hanno dimostrato che l'associazione di farmaci antiaggreganti piastrinici, sebbene riduca il rischio di tromboembolismo rispetto all'ASA da solo, non riduce il rischio di tromboembolismo cerebrale e/o sistemico rispetto ai farmaci VKA [20], determinando un aumento significativo delle emorragie sistemiche sia maggiori sia totali rispetto all'ASA da solo [21], con un tasso simile a quello dei VKA. È peraltro d'obbligo sottolineare come le differenze che emergono dallo studio ACTIVE-W siano particolarmente significative a favore di warfarin solo nei pazienti con TTR $>65 \%$, mentre si riducono nettamente nei pazienti con TTR $<65 \%$ [22]. Il beneficio netto apportato dall'aggiunta di clopidogrel all'ASA potrebbe non essere significativo rispetto all'ASA da solo quando vengono valutati come endpoint la mortalità o l'associazione mortalità/disabilità dopo stroke ischemico o emorragico [23].

Le linee guida delle più importanti Società Scientifiche Internazionali - ESC, American College of Cardiology (ACC), American Heart Association (AHA), Canadian Cardiovascular Society (CCS), American College of Chest Physicians (ACCP) $[1-3,24]$ - e le linee guida italiane SPREAD sulla gestione dell'ictus [25] sono concordi nell'indicare i farmaci VKA come prima scelta nella profilassi primaria e secondaria dell'ictus nei pazienti con FA ad alto rischio tromboembolico $\left(\mathrm{CHADS}_{2} \geq 2\right)$. Nei pazienti con $\mathrm{CHADS}_{2}$ 0-1 o laddove occorra stimare meglio il rischio embolico, l'ESC suggerisce di calcolare il rischio con il $\mathrm{CHA}_{2} \mathrm{DS}_{2}$-VASC [1]. Se il punteggio è $\geq 2$ viene indicata la terapia con VKA, se è pari a 1 l'ESC indica VKA o ASA con lo stesso grado di raccomandazione, ma suggerisce di preferire i farmaci VKA. Infine, se il punteggio $\mathrm{CHA}_{2} \mathrm{DS}_{2}$-VASC è pari a 0 viene consigliata terapia con ASA 75$325 \mathrm{mg}$ oppure nessuna terapia, e quest'ultima opzione è da preferire in assenza di altri fattori di rischio [1]. L'associazione di ASA-clopidogrel al momento è consigliata, con basso grado di raccomandazione (IIB), solo dalle linee guida americane sul management della FA, recentemente aggiornate [26], in alternativa ai farmaci VKA nei pazienti a basso rischio di sanguinamento.

Nonostante negli ultimi anni sia notevolmente cresciuta la prescrizione dei farmaci VKA in questo contesto, tali farmaci presentano importanti limitazioni che ne condizionano l'uso e l'ulteriore diffusione, tanto da essere una classe farmacologica ancora nettamente sottoutilizzata nonostante le solide evidenze scientifiche [27-29]. Una metanalisi recentemente pubblicata [27] dimostra che meno del $60 \%$ dei pazienti con FA ad alto rischio di eventi tromboembolici è in trattamento con farmaci VKA. In Italia, studi clinici dimostrano che oltre il 50\% dei pazienti con FA ad alto rischio tromboembolico non è trattato o è trattato con ASA o con VKA ma mantenendo valori di INR $<2,0$ [28]. Altri studi evidenziano che la percentuale di pazienti affetti da FA che assumono farmaci VKA prima di un ictus cardioembolico oscilla tra il 13\% [29] e il 27,2\% [30], molti dei quali in range terapeutico non ottimale [29]. Sebbene i trial clinici dimostrino come gli eventi ischemici cerebrali si verifichino in pazienti con valori di INR inferiori al range terapeutico [31], è da sottolineare che una percentuale non trascurabile di pazienti che assumono farmaci VKA sviluppa eventi ischemici cerebrali nonostante una condotta terapeutica ottimale [32].

I fattori che possono determinare il sottoutilizzo dei farmaci VKA sono molteplici e legati alla loro scarsa maneggevolezza (multiple interazioni farmacologiche, alla variabilità genetica del metabolismo, alla necessità di un tempo di induzione per raggiungere l'obiettivo terapeutico), alla scarsa aderenza alla terapia da parte dei pazienti (necessità di monitoraggio laboratoristico frequente) nonché al basso indice terapeutico (rischio di emorragia) [33-36].

\section{La valutazione del rischio emorragico nella fibrillazione atriale}

I principali fattori che possono determinare un sanguinamento in corso di terapia con farmaci VKA sono l'intensità dell'effetto anticoagulante, le caratteristiche intrinseche del paziente e la durata del periodo di trattamento [34]. Sebbene esistano differenti scale prognostiche per predire il rischio di emorragia, come l'HAS-BLED o l'Anticoagulation and Risk Factors in Atrial Fibrillation (ATRIA) recentemente proposti (Allegato 2) [1,37], è da notare che molti fattori che predicono un aumentato rischio di emorragia sono allo stesso tempo fattori predittivi di un aumentato rischio tromboembolico, per cui risulta difficile nel singolo paziente decidere quale sia la giusta terapia e pesare concretamente il rapporto rischio/beneficio nella scelta terapeutica.

Inoltre, queste scale prognostiche non dovrebbero essere utilizzate come il solo criterio per decidere se iniziare o meno la terapia con farmaci VKA, ma dovrebbero essere comprese in una valutazione complessiva del paziente che tenga conto dello stato funzionale e cognitivo, della verosimile aderenza alla terapia, del rischio tromboembolico e della preferenza personale. Clinicamente è possibile usare questi modelli per valutare "a priori" i rischi e i benefici della terapia con farmaci VKA, aggiustando potenzialmente la dose, il tipo, la durata o la frequenza del monitoraggio INR. Tali valutazioni possono essere effettuate all'inizio della terapia e ripetute periodicamente nel corso della terapia.

In futuro, l'utilizzo di test genetici in grado di valutare il metabolismo epatico dei farmaci VKA, come per esempio la tipizzazione dei polimorfismi del citocromo $\mathrm{P} 450$, potrà aiutare a identificare quei pazienti più facilmente predisposti a complicanze emorragiche in corso di terapia con farmaci VKA.

\section{I nuovi farmaci anticoagulanti: gli inibitori diretti della trombina (dabigatran) e del fattore Xa (rivaroxaban, apixaban)}

Al fine di superare le predette limitazioni dei farmaci VKA, negli ultimi anni la ricerca farmacologica e clinica ha portato avanti lo studio e la sperimentazione clinica di nuove molecole attive sulla coagulazione, somministrabili per via orale, che potessero raggiungere almeno gli stessi risultati, rispetto ai farmaci VKA, in termini di efficacia nella prevenzione tromboembolica, con profili di sicurezza equivalenti [38]. Dopo il fallimento di ximelagatran - un anticoagulante orale inibitore diretto della trombina, immesso in commercio in 
virtù degli ottimi risultati in termini di efficacia antitrombotica, ma successivamente ritirato a causa dell'epatotossicità - negli ultimi anni nuovi anticoagulanti orali hanno raggiunto la sperimentazione clinica di fase III [38-42]. Grazie ai risultati positivi di efficacia e sicurezza nella profilassi del tromboembolismo venoso nei pazienti sottoposti a interventi di artroprotesi d'anca e di ginocchio, i nuovi farmaci anticoagulanti orali inibitori diretti della trombina (dabigatran) e del fattore Xa (rivaroxaban, apixaban) sono stati recentemente confrontati con il warfarin in trial randomizzati e controllati (RCT) di fase III nella profilassi tromboembolica del paziente con FA non valvolare, con l'obiettivo di dimostrarne la non inferiorità in termini di efficacia e sicurezza rispetto al warfarin [39-42]. Le proprietà farmacocinetiche e dinamiche dei nuovi anticoagulanti orali sono riassunte nell'Allegato 3 [36].

I risultati degli RCT RE-LY (Randomized Evaluation of Long Term Anticoagulation Therapy) per dabigatran $[39,40]$, ROCKET-AF (Rivaroxaban Once Daily Oral Direct Factor Xa Inhibition Compared with Vitamin K Antagonism for Prevention of Stroke and Embolism Trial in Atrial Fibrillation) per rivaroxaban [41] e ARISTOTLE (Apixaban for Reduction in Stroke and Other Thromboembolic Events in Atrial Fibrillation) per apixaban [42] sono stati recentemente pubblicati. Per tutti e tre i farmaci (dabigatran, rivaroxaban e apixaban), confrontati con warfarin, è stato raggiunto l'obiettivo principale di non inferiorità in maniera statisticamente significativa, in presenza di un ottimo profilo di sicurezza [39-42]

\section{Gli studi RE-LY, ROCKET-AF e ARISTOTLE}

\section{Disegno, criteri di inclusione ed esclusione, definizione degli outcome, caratteristiche generali}

Gli studi RE-LY, ROCKET-AF e ARISTOTLE sono tre trial clinici multicentrici, randomizzati, controllati che hanno valutato come obiettivo primario la non inferiorità in termini di efficacia e sicurezza di tre molecole ad azione anticoagulante orale - rispettivamente dabigatran, rivaroxaban e apixaban - nella profilassi antitrombotica dei pazienti con FA parossistica o persistente/permanente nei confronti del warfarin, il più diffuso farmaco VKA [39-42]. Gli studi ROCKET-AF e ARISTOTLE hanno anche valutato l'ipotesi della superiorità, rispettivamente, di rivaroxaban per il profilo di sicurezza durante la fase di trattamento (intention to treat) e apixaban, per il profilo sia di efficacia sia di sicurezza, nei confronti del warfarin [41,42]. All'interno del protocollo dello studio RE-LY, una volta dimostrata la non inferiorità, ci si prefiggeva inoltre di analizzare le eventuali superiorità. Lo studio RE-LY prevedeva la somministrazione in doppio cieco solo dei differenti dosaggi di dabigatran, mentre la somministrazione del warfarin avveniva in aperto $[39,40]$. Gli studi ROCKET-AF e ARISTOTLE prevedevano la somministrazione in doppio cieco sia del farmaco di confronto sia del warfarin $[41,42]$. Nei tre RCT il warfarin è stato somministrato a un dosaggio tale da mantenere i valori di INR nel range terapeutico raccomandato nei pazienti con FA non valvolare, cioè 2.0-3.0, con target 2.5 [39-42].
I tre RCT hanno confrontato complessivamente più di 28.000 pazienti trattati con i nuovi anticoagulanti orali (28.272) con oltre 22.000 pazienti trattati con warfarin (22.184) [39-42]. La differenza numerica è ascrivibile allo studio RE-LY, in cui sono stati confrontati tre bracci di trattamento di cui 2 trattati con dabigatran a dosaggi differenti (110 mg bis/die e $150 \mathrm{mg}$ bis/die) e uno con warfarin $[39,40]$. Tutti e tre gli RCT hanno confrontato popolazioni omogenee di pazienti [39-42].

Il disegno dei tre RCT, con i criteri di inclusione ed esclusione, è riassunto in tabella 1 [39-42].

Il disegno dello studio RE-LY prevedeva, come già sottolineato, due dosaggi di dabigatran $[39,40]$.

Quello dello studio ROCKET-AF prevedeva un dosaggio differenziato di rivaroxaban, ridotto nei pazienti con insufficienza renale moderata $(20 \mathrm{mg}$ in monosomministrazione nel paziente non nefropatico e $15 \mathrm{mg}$ nel nefropatico) [41].

Nello studio ARISTOTLE il dosaggio di apixaban era pari a $5 \mathrm{mg}$ bis/die per la grande maggioranza di pazienti e a 2,5 mg bis/die nel 4,7\% dei pazienti che presentavano almeno due delle seguenti caratteristiche: età $\geq 80$ anni, peso $<60 \mathrm{~kg}$, creatinina $>1,5 \mathrm{mg} / \mathrm{dL}$ [42].

I criteri di inclusione ed esclusione appaiono quindi sostanzialmente simili nello studio $\operatorname{RE}-\operatorname{LY}[39,40]$ e nello studio ARISTOTLE [42], mentre sono in parte differenti nel ROCKET-AF [41]. La tabella 2 riassume i criteri utilizzati per la definizione del profilo di sicurezza emorragico. Nello studio RE-LY si differenziano le emorragie maggiori da quelle minori, peraltro scorporando nelle maggiori il sottogruppo delle emorragie a rischio di vita (life-threatening) $[39,40]$; negli studi ROCKET-AF e ARISTOTLE sono state analizzate le emorragie maggiori, le emorragie non maggiori ma clinicamente rilevanti e le emorragie minori $[41,42]$.

Gli obiettivi primari di efficacia e sicurezza appaiono analoghi nei tre RCT [39-42] (tab. 1). L'obiettivo primario di efficacia era rappresentato dalla somma degli ictus ischemici ed emorragici e degli eventi tromboembolici sistemici. Per quanto riguarda l'obiettivo primario di sicurezza, esso era rappresentato dagli eventi emorragici maggiori; tuttavia nel ROCKET-AF è stata considerata la somma degli eventi emorragici maggiori e dei non maggiori ma clinicamente rilevanti [41].

La tabella 3 sintetizza le caratteristiche generali dei pazienti inclusi nei tre RCT [39-42]. Esiste una sostanziale analogia tra di essi in termini di età, sesso, durata del followup e alcuni fattori di rischio, tuttavia è da sottolineare che nello studio ROCKET-AF il $100 \%$ dei pazienti presenta un punteggio $\mathrm{CHADS}_{2} \geq 2$ [41], mentre negli studi RE-LY e ARISTOTLE le percentuali dei pazienti con punteggio $\mathrm{CHADS}_{2}$ $\geq 2$ è, rispettivamente, del $67 \%$ e del $66 \%$ [39-42]. Nello studio ROCKET-AF l' $87 \%$ dei pazienti ha un punteggio $\mathrm{CHADS}_{2}$ $\geq 3$ [41] rispetto al $32,6 \%$ e al $30,2 \%$ degli studi RE-LY e ARISTOTLE $[39,40]$. Tali differenze sono presumibilmente imputabili alla più elevata percentuale di pazienti con anamnesi di pregresso TIA/ictus ischemico/tromboembolismo sistemico $(54,9 \%$ vs $20 \%$ nel RE-LY e vs $19,2 \%$ nell'ARISTOTLE) o di pazienti affetti da scompenso cardiaco presenti nel ROCKET-AF $\quad(62,6 \%$ vs $32 \%$ nel RE-LY e vs $35,5 \%$ nell'ARISTOTLE [39-42]. Inoltre la percentuale di pazienti con FA parossistica appare significativamente maggiore nel 
Tabella 1 Disegno, criteri di selezione e outcome primari di efficacia e sicurezza dei pazienti arruolati nei tre RCT.

\begin{tabular}{|c|c|c|}
\hline \multicolumn{3}{|c|}{ Disegno dello studio } \\
\hline RE-LY (dabigatran) & ROCKET-AF (rivaroxaban) & ARISTOTLE (apixaban) \\
\hline $\begin{array}{l}110 \mathrm{mg} 2 \text { volte/die } \\
0 \\
150 \mathrm{mg} 2 \text { volte/die } \\
\text { vs } \\
\text { warfarin INR 2,0-3,0 }\end{array}$ & $\begin{array}{l}20 \mathrm{mg} 1 \text { volta/die (15 mg } 1 \text { volta/die nei } \\
\text { pazienti con insufficienza renale moderata, } \\
\text { clearance della creatinina } 30-50 \mathrm{~mL} / \mathrm{min}) \\
\text { vs } \\
\text { warfarin INR } 2,0-3,0\end{array}$ & $\begin{array}{l}5 \mathrm{mg} 2 \text { volte/die }(2,5 \mathrm{mg} 2 \text { volte } / \text { die in } \\
\text { pazienti con almeno due delle seguenti } \\
\text { caratteristiche: età } \geq 80 \text { anni, peso }<60 \mathrm{~kg} \text {, } \\
\text { creatinina }>1,5 \mathrm{mg} / \mathrm{dL}) \\
\text { vs } \\
\text { warfarin INR 2,0-3,0 }\end{array}$ \\
\hline \multicolumn{3}{|c|}{ Criteri } \\
\hline & Inclusione & Esclusione \\
\hline $\begin{array}{l}\text { RE-LY } \\
\text { (dabigatran) }\end{array}$ & $\begin{array}{l}\text { FA documentata all'ECG di screening o nei } \\
\text { precedenti } 6 \text { mesi + almeno uno tra: } \\
\text { - pregresso ictus o TIA } \\
\text { - FE }<40 \% \\
\text { - Classe NYHA } \geq 2 \text { entro } 6 \text { mesi prima dello } \\
\text { screening } \\
\text { - età } \geq 75 \text { anni o } \\
\text { - età } 65-74 \text { anni + diabete mellito, } \\
\text { ipertensione arteriosa o malattia } \\
\text { coronarica }\end{array}$ & $\begin{array}{l}\text { Età }<18 \text { anni } \\
\text { Valvulopatia di grado severo } \\
\text { Ictus nelle precedenti } 2 \text { settimane o ictus } \\
\text { severo entro } 6 \text { mesi } \\
\text { Condizioni predisponenti eventi emorragici } \\
\text { Insufficienza renale severa (clearance della } \\
\text { creatinina }<30 \mathrm{~mL} / \mathrm{min} \text { ) } \\
\text { Malattia epatica attiva } \\
\text { Gravidanza }\end{array}$ \\
\hline $\begin{array}{l}\text { ROCKET-AF } \\
\text { (rivaroxaban) }\end{array}$ & $\begin{array}{l}\text { FA documentata all'ECG + almeno uno tra: } \\
\text { - pregresso ictus o TIA o embolia sistemica } \\
\text { o almeno due tra: } \\
\text { - scompenso cardiaco o FE } \leq 35 \% \text {, } \\
\text { ipertensione arteriosa, diabete mellito, } \\
\text { età } \geq 75 \text { anni }\end{array}$ & $\begin{array}{l}\text { Età }<18 \text { anni } \\
\text { Stenosi mitralica significativa } \\
\text { FA parossistica da causa reversibile } \\
\text { Sanguinamento interno attivo } \\
\text { Ictus con severa dosabilità } \\
\text { Anamnesi di emorragia intracranica } \\
\text { Patologie emorragiche } \\
\text { Insufficienza renale severa (clearance della } \\
\text { creatinina }<30 \mathrm{~mL} / \mathrm{min} \text { ) }\end{array}$ \\
\hline $\begin{array}{l}\text { ARISTOTLE } \\
\text { (apixaban) }\end{array}$ & $\begin{array}{l}\text { FA documentata all'ECG in } 2 \text { settimane non } \\
\text { consecutive nei } 12 \text { mesi precedenti + almeno } \\
\text { uno tra: } \\
\text { - età } \geq 75 \text { anni } \\
\text { - pregresso ictus o TIA o embolia sistemica } \\
\text { - scompenso cardiaco nei precedenti } 3 \text { mesi o } \\
\text { FE } \leq 40 \% \\
\text { - diabete mellito } \\
\text { - ipertensione arteriosa }\end{array}$ & $\begin{array}{l}\text { Età }<18 \text { anni } \\
\text { FA da cause reversibili } \\
\text { Stenosi mitralica moderato-severa } \\
\text { Condizioni necessitanti anticoagulazione } \\
\text { (es. protesi valvolari) } \\
\text { Ictus nella settimana precedente } \\
\text { Condizioni richiedenti un dosaggio di ASA } \\
>165 \mathrm{mg} \text { oppure richiedenti } \\
\text { ASA + clopidogrel } \\
\text { Insufficienza renale severa (creatinina } \\
>2,5 \mathrm{mg} / \mathrm{dL} \text { o clearance della creatinina } \\
<25 \mathrm{~mL} / \mathrm{min} \text { ) }\end{array}$ \\
\hline
\end{tabular}

\begin{tabular}{lcc}
\hline & \multicolumn{1}{c}{ Outcome primario di efficacia } & ARISTOTLE (apixaban) \\
\hline $\begin{array}{l}\text { RE-LY (dabigatran) } \\
\begin{array}{l}\text { Somma di ictus ischemici } \\
\text { tromboembolici sistemici }\end{array}\end{array}$ & $\begin{array}{l}\text { Somma di ictus ischemici ed emorragici ed } \\
\text { eventi tromboembolici sistemici }\end{array}$ & $\begin{array}{l}\text { Somma di ictus ischemici ed emorragici ed } \\
\text { eventi tromboembolici sistemici }\end{array}$ \\
\hline \multicolumn{1}{c}{ Outcome primario di sicurezza } & \\
\hline RE-LY (dabigatran) & ROCKET-AF (rivaroxaban) & ARISTOTLE (apixaban) \\
\hline Emorragie maggiori & $\begin{array}{l}\text { Somma di emorragie maggiori e non maggiori } \\
\text { ma clinicamente rilevanti }\end{array}$ & Emorragie maggiori \\
\hline
\end{tabular}

RE-LY (32,4\% vs 17,5\% nel ROCKET-AF e vs 15,1\% nell'ARISTOTLE), mentre è minore la percentuale di pazienti ipertesi nello stesso studio rispetto agli altri [39-42]. Altri due aspetti, infine, sono da sottolineare (tab. 3): la pregressa terapia con ASA variabile dal 31,3\% dell'ARISTOTLE al 36,3\% del ROCKET-AF al 39\% del RE-LY all'inizio dello studio e la media trascorsa nel TTR, variabile dal 55\% del ROCKET-AF al 62,2\% dell'ARISTOTLE al 64\% del RE-LY 
Tabella 2 Definizione degli eventi emorragici nei tre RCT.

\begin{tabular}{|c|c|c|c|}
\hline & RE-LY (dabigatran) & ROCKET-AF (rivaroxaban) & ARISTOTLE (apixaban) \\
\hline $\begin{array}{l}\text { Emorragia } \\
\text { maggiore }\end{array}$ & $\begin{array}{l}\text { Calo dell'emoglobina di almeno } \\
2 \mathrm{~g} / \mathrm{L} \\
\text { Emorragia richiedente almeno } \\
2 \text { unità } \\
\text { di eritrociti concentrati o } \\
\text { sangue intero } \\
\text { Emorragia sintomatica di un } \\
\text { organo critico } \\
\text { Emorragia life-threatening, } \\
\text { sottogruppo } \\
\text { dell'emorragia maggiore } \\
\text { caratterizzato da: } \\
\text { - emorragia fatale } \\
\text { - emorragia intracranica } \\
\text { sintomatica } \\
\text { - calo dell'emoglobina di } \\
\text { almeno } 5 \text { g/L } \\
\text { - emorragia richiedente almeno } \\
4 \text { unità di eritrociti concentrati } \\
\text { o sangue intero o inotropi } \\
\text { o intervento chirurgico }\end{array}$ & $\begin{array}{l}\text { Emorragia fatale } \\
\text { Calo dell'emoglobina di almeno } \\
2 \mathrm{~g} / \mathrm{L} \\
\text { Emorragia richiedente almeno } \\
2 \text { unità di eritrociti concentrati } \\
\text { o sangue intero } \\
\text { Emorragia sintomatica di un } \\
\text { organo critico (cervello, midollo } \\
\text { spinale, occhio, torace, } \\
\text { pericardio, peritoneo, } \\
\text { retroperitoneo, articolazioni, } \\
\text { tratto gastroenterico, } \\
\text { intramuscolare con sindrome } \\
\text { compartimentale) }\end{array}$ & $\begin{array}{l}\text { Emorragia fatale } \\
\text { Calo dell'emoglobina } \\
\text { di almeno } 2 \mathrm{~g} / \mathrm{L} \\
\text { Emorragia richiedente } \\
\text { almeno } 2 \text { unità di } \\
\text { eritrociti concentrati o } \\
\text { sangue intero } \\
\text { Emorragia sintomatica di } \\
\text { un organo critico } \\
\text { (cervello, midollo } \\
\text { spinale, occhio, torace, } \\
\text { peritoneo, } \\
\text { retroperitoneo, } \\
\text { articolazioni, tratto } \\
\text { gastroenterico) }\end{array}$ \\
\hline $\begin{array}{l}\text { Emorragia non } \\
\text { maggiore ma } \\
\text { clinicamente } \\
\text { rilevante }\end{array}$ & Non considerata & $\begin{array}{l}\text { Emorragie evidenti non } \\
\text { classificabili come maggiori ma } \\
\text { richiedenti intervento medico, } \\
\text { temporanea cessazione del } \\
\text { farmaco o ritardo nella } \\
\text { successiva somministrazione, } \\
\text { determinante dolore o } \\
\text { impedimenti nelle attività della } \\
\text { vita quotidiana }\end{array}$ & $\begin{array}{l}\text { Emorragia richiedente: } \\
\text { - ospedalizzazione } \\
\text { - trattamento } \\
\text { farmacologico o } \\
\text { chirurgico gestito dal } \\
\text { medico } \\
\text { - cambiamento } \\
\text { farmacologico della } \\
\text { terapia antitrombotica }\end{array}$ \\
\hline $\begin{array}{l}\text { Emorragia } \\
\text { minore }\end{array}$ & Tutte le altre & Tutte le altre & Tutte le altre \\
\hline
\end{tabular}

Tabella 3 Caratteristiche generali della popolazione dei tre RCT.

\begin{tabular}{llll}
\hline & RE-LY (dabigatran) & ROCKET-AF (rivaroxaban) & ARISTOTLE (apixaban) \\
\hline Età (anni) & $71,5 \pm 8,5$ (media) & 73 (mediana) & 70 (mediana) \\
\hline Rapporto M/F (\%) & $64 / 36$ & $60,3 / 39,7$ & $64,5 / 35,5$ \\
\hline Follow-up (mesi) & 30 & 28 & 30 \\
\hline TTR (\%, media) & 64 & 55 & 62,2 \\
\hline CHADS $_{2} \geq 2$ (\%) & 67 & 100 & 66 \\
\hline CHADS $2 \geq 3$ (\%) & 32,6 & 87 & 30,2 \\
\hline Pregresso ictus/TIA (\%) & 20 & 54,9 (pregresso ictus/ & 19,2 \\
\hline ASA al basale (\%) & & TIA/embolia sistemica) & 31,3 \\
\hline Pregresso infarto miocardico acuto (\%) & 16,8 & 36,3 & 14,5 \\
\hline Ipertensione arteriosa (\%) & 78,8 & 16,6 & 87,3 \\
\hline Diabete mellito (\%) & 23,2 & 90,3 & 25,0 \\
\hline Scompenso cardiaco (\%) & 32,0 & 40,4 & 35,5 \\
\hline FA parossistica (\%) & 32,4 & 62,6 & 15,1 \\
\hline FA permanente/persistente (\%) & 67,6 & 17,5 & 84,9
\end{tabular}




\section{Risultati di efficacia}

Tutti e i tre gli RCT hanno raggiunto l'obiettivo principale di efficacia. Dabigatran (110 mg bis/die: RR 0,90, IC $95 \% 0,74-$ $1,10, \mathrm{p}<0,001 ; 150 \mathrm{mg}$ bis/die: RR 0,66, IC 95\% 0,52-0,81, $\mathrm{p}<0,001)$, rivaroxaban (HR 0,79, IC 95\% 0,66-0,96, $\mathrm{p}<0,001$ ) e apixaban (HR 0,79, IC 95\% 0,66-0,95, p=0,01) risultano significativamente non inferiori al warfarin nella prevenzione dell'ictus ischemico e degli eventi tromboembolici nel paziente con FA non valvolare senza aumentare il rischio di ictus emorragico. Il rischio di tali eventi risulta rispettivamente dell' $1,54 \%, 1,11 \%, 1,7 \%$ e $1,27 \%$ per anno di trattamento per dabigatran $110 \mathrm{mg}$ bis/die e $150 \mathrm{mg}$ bis/die, rivaroxaban e apixaban rispetto all'1,71\%, 2,2\%, 1,60\% per warfarin negli studi RE-LY, ROCKET-AF e ARISTOTLE [39-42]. Dabigatran $150 \mathrm{mg}$ bis/die, rivaroxaban e apixaban risultano superiori al warfarin in questo contesto. Il dosaggio di dabigatran $150 \mathrm{mg}$ bis/die risulta superiore al warfarin nella prevenzione degli ictus ischemici (RR 0,76, IC 95\% 0,59-0,97, $p=0,03$ ), mentre dabigatran $110 \mathrm{mg}$ bis/die, rivaroxaban e apixaban risultano non inferiori al warfarin nella profilassi dell'ictus ischemico.

La figura 1 mostra la riduzione del rischio relativo (RRR) degli eventi tromboembolici emersi nei tre RCT. Complessivamente i nuovi anticoagulanti orali riducono il rischio relativo di eventi embolici cerebrali e sistemici e gli ictus emorragici di circa il $20 \%$. Gli eventi ischemici cerebrali disabilitanti e fatali risultano significativamente inferiori per dabigatran $150 \mathrm{mg}$ bis/die e apixaban rispetto al warfarin (il dato non è riportato per rivaroxaban), mentre non risultano differenze significative tra warfarin e dabigatran $100 \mathrm{mg}$ bis/die.

I tre RCT evidenziano inoltre una RRR complessiva del 7,5\% per tutte le cause di mortalità associata ai nuovi farmaci anticoagulanti orali (fig. 2), seppur solo ai limiti della significatività statistica per apixaban (HR 0,89, IC 95\% 0,81$0,98, \mathrm{p}=0,047$ ). Dabigatran $150 \mathrm{mg}$ bis/die è superiore al warfarin nella riduzione della mortalità per cause vascolari (RR 0,85, IC 95\% 0,72-0,99, p= 0,04); dabigatran $110 \mathrm{mg}$ bis/ die, rivaroxaban e apixaban risultano non inferiori al warfarin in questo contesto.

\section{Risultati di sicurezza}

I tre RCT dimostrano che i nuovi anticoagulanti orali non sono inferiori al warfarin nel profilo di sicurezza. Dabigatran al dosaggio di $110 \mathrm{mg}$ bis/die e apixaban risultano significativamente superiori al warfarin nella riduzione degli eventi emorragici totali (nel RE-LY emorragie maggiori e minori, nel ROCKET-AF e in ARISTOTLE emorragie maggiori e non maggiori clinicamente rilevanti), maggiori e intracranici, mentre al dosaggio di $150 \mathrm{mg}$ bis/die dabigatran riduce significativamente solo gli eventi emorragici minori e quelli intracranici, ma non quelli maggiori [39-42]. Rivaroxaban riduce significativamente le emorragie intracraniche, ma non presenta significative differenze rispetto al warfarin nello sviluppo di eventi emorragici maggiori e nella somma di emorragie maggiori e non maggiori clinicamente rilevanti [41]. La figura 3 riporta il rischio per 100 pazienti/anno di eventi emorragici totali, maggiori e intracranici nei tre RCT, mentre la figura 4 evidenzia la RRR, rispettivamente, di emorragie totali, maggiori ed emorragie intracraniche. Dabigatran $110 \mathrm{mg}$ bis/die, rivaroxaban e apixaban sono superiori al warfarin nel ridurre le emorragia fatali. Dabigatran al dosaggio di $150 \mathrm{mg}$ bis/die ( RR 1,48 , IC 95\% $1,18-1,85, \mathrm{p}=0,001)$ e rivaroxaban $(3,2 \%$ vs $2,2 \%, p<0,001)$ aumentano significativamente il rischio di emorragie gastrointestinali. La tabella 4 riassume i principali risultati di efficacia e sicurezza emersi nei tre RCT.

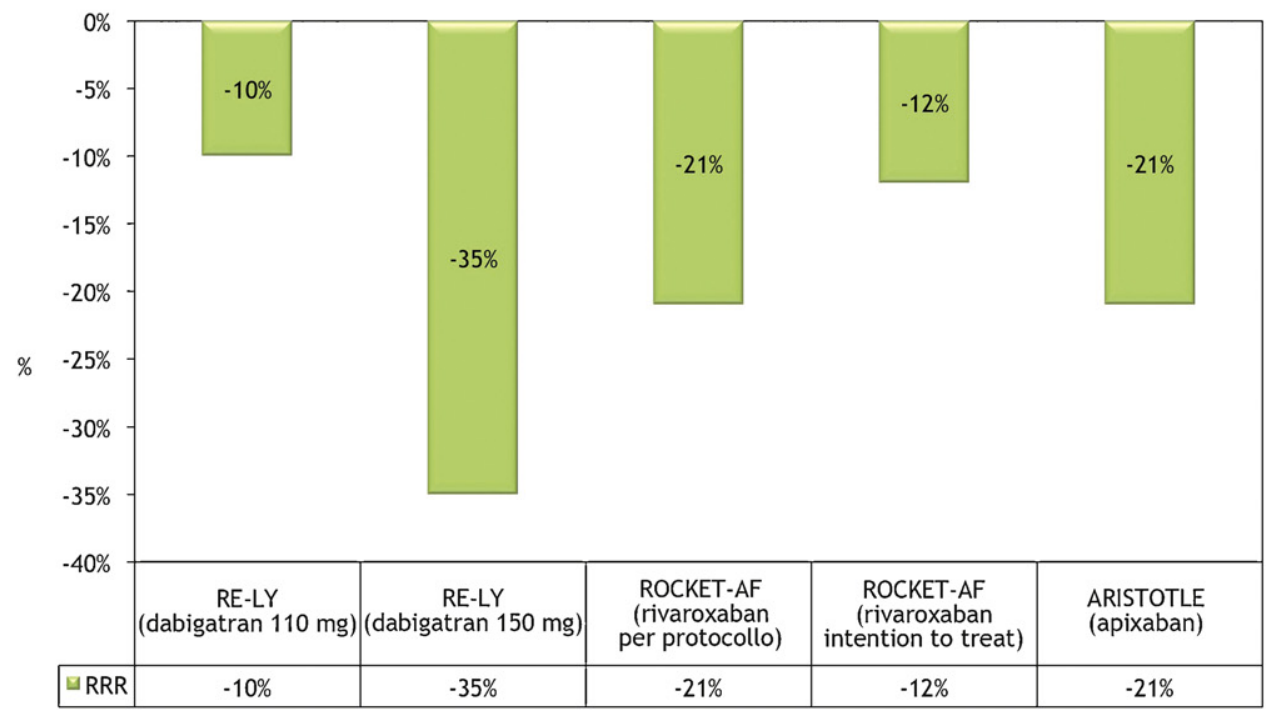

Figura 1 Riduzione del rischio relativo (RRR) di ictus ischemico ed emorragico e tromboembolismo sistemico dei nuovi anticoagulanti orali rispetto al warfarin. 


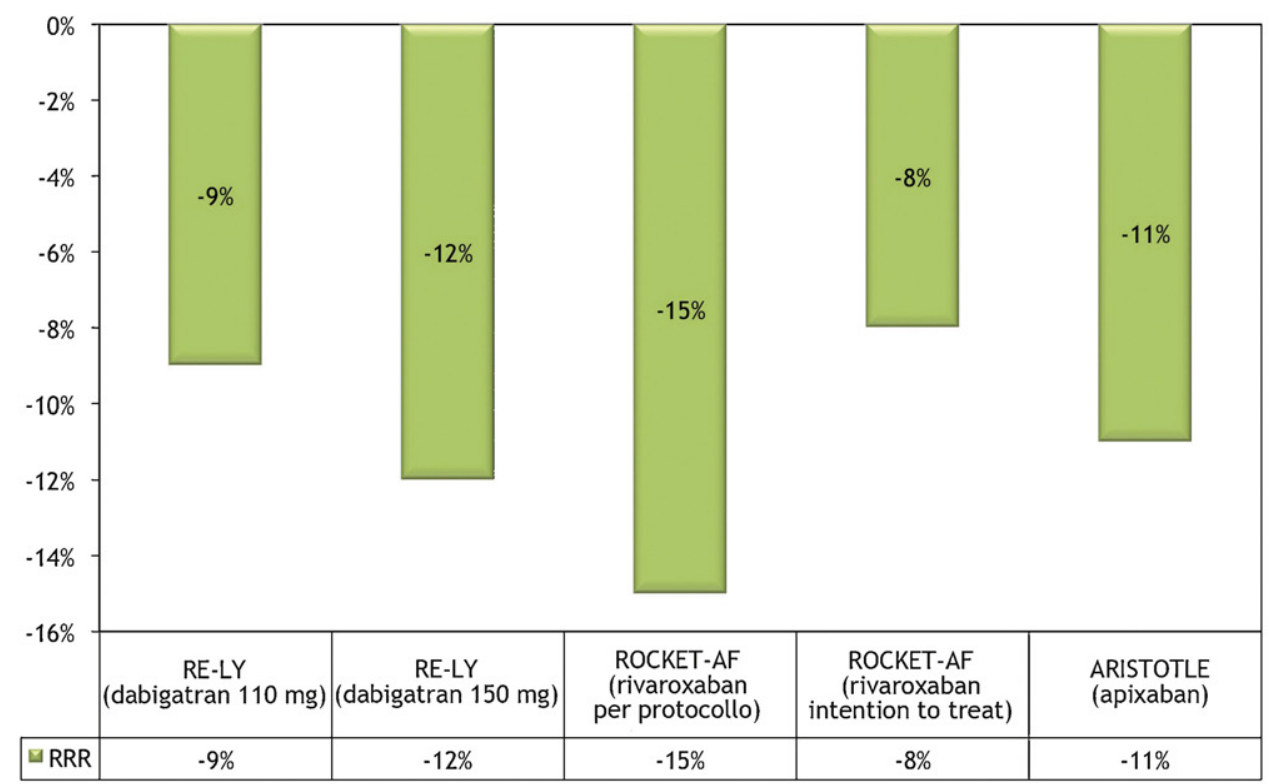

Figura 2 Riduzione del rischio relativo (RRR) di mortalità per tutte le cause dei nuovi anticoagulanti orali rispetto al warfarin.

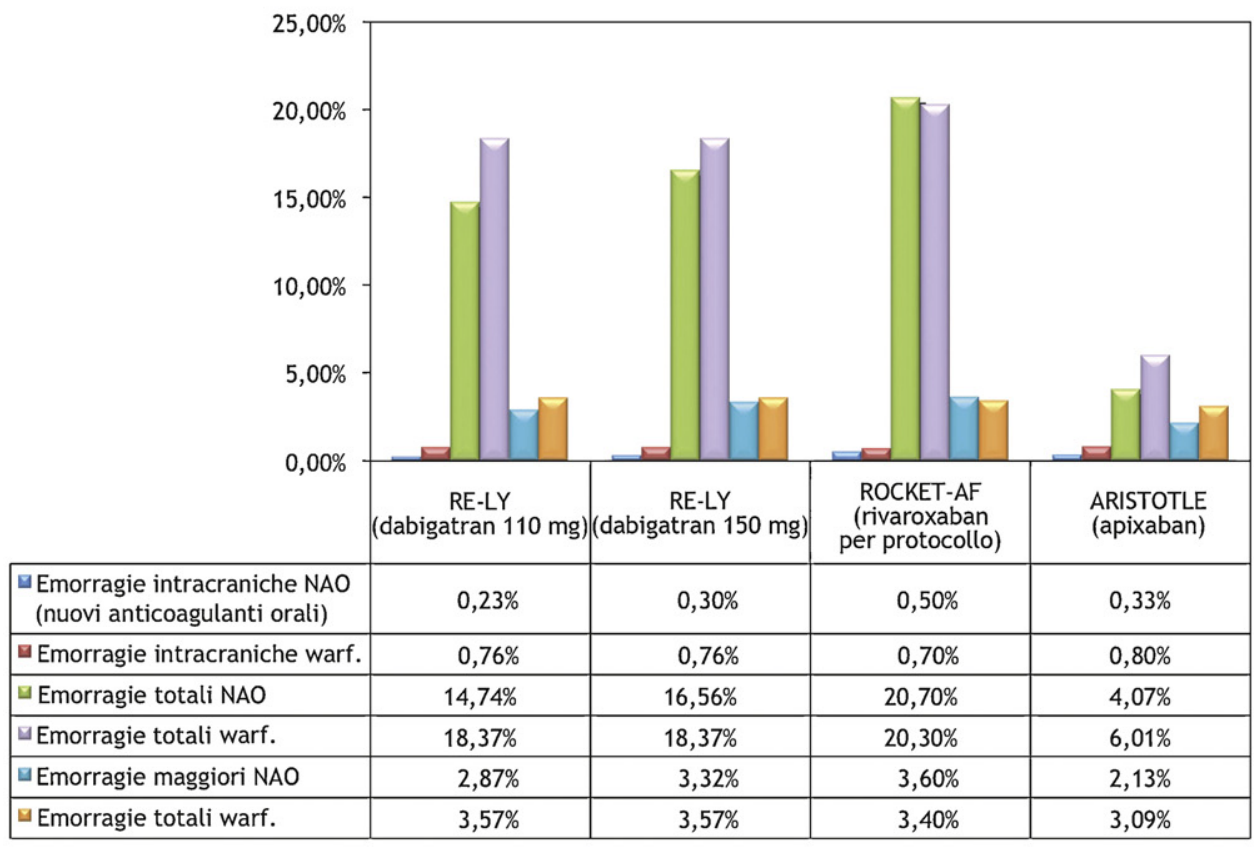

Figura 3 Incidenza di emorragie intracraniche, totali e maggiori per 100 pazienti/anno di trattamento nei tre RCT.

\section{Discussione}

I risultati degli RCT che hanno confrontato tre nuove molecole ad azione anticoagulante orale con il warfarin raggiungono l'obiettivo della dimostrazione di non inferiorità in termini di efficacia e sicurezza nella profilassi antitromboembolica del paziente con FA non valvolare. Per alcuni endpoint analizzati, peraltro, i nuovi anticoagulanti orali sembrano essere superiori ai farmaci VKA. In particolare, gli studi di fase III dimostrano la superiorità dei nuovi anticoagulanti orali rispetto al warfarin nel prevenire l'endpoint combinato eventi tromboembolici ischemici (cerebrali e sistemici) ed emorragici cerebrali. 


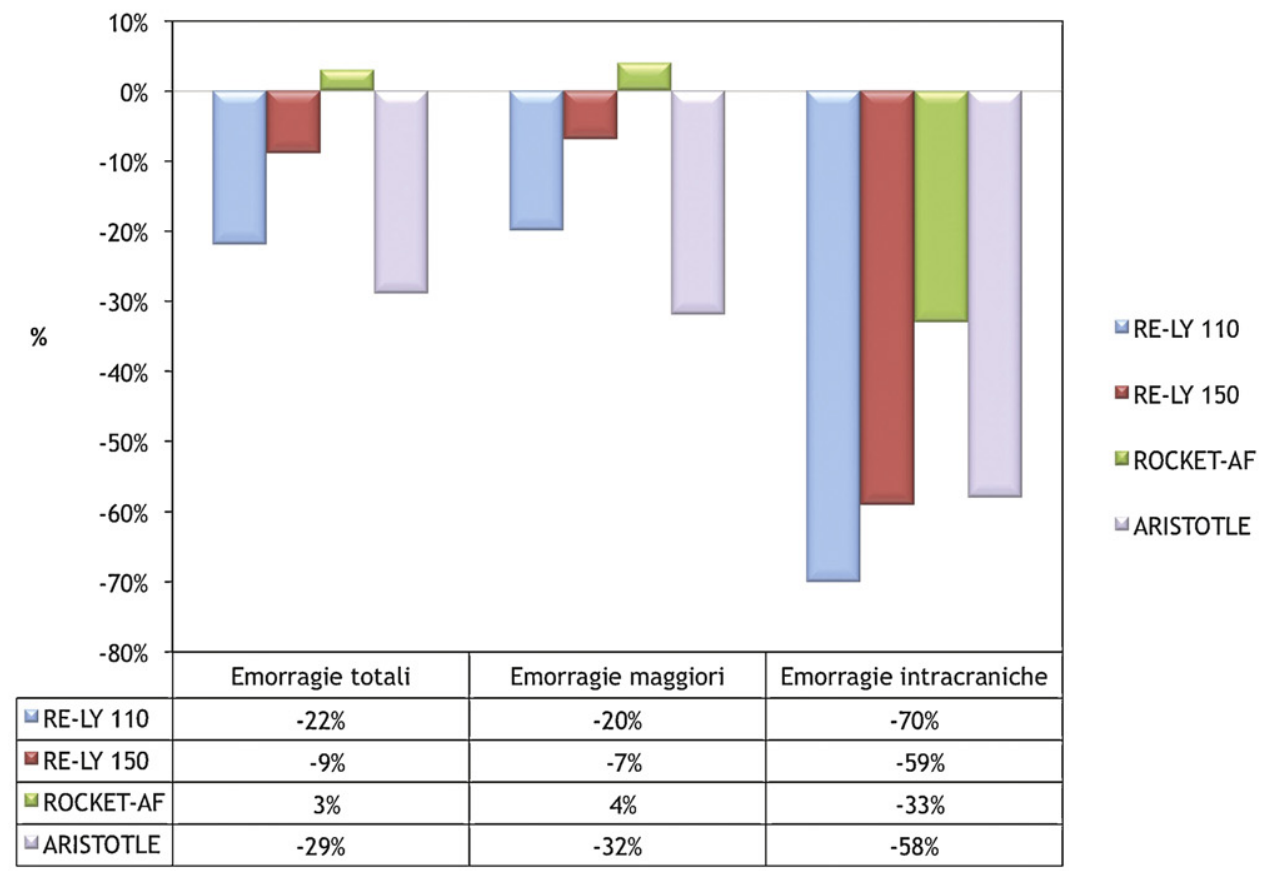

Figura 4 Riduzione del rischio relativo (RRR) di eventi emorragici totali, maggiori e intracranici dei nuovi anticoagulanti orali rispetto al warfarin.

Tabella 4 Sintesi dei principali risultati emersi negli studi di confronto tra nuovi farmaci anticoagulanti orali e warfarin.

\begin{tabular}{|c|c|c|c|c|}
\hline & $\begin{array}{l}\text { RE-LY } 110 \mathrm{mg} \\
\text { RR (IC 95\%I) }\end{array}$ & $\begin{array}{l}\text { RE-LY } 150 \mathrm{mg} \\
\text { RR (IC 95\%) }\end{array}$ & $\begin{array}{l}\text { ROCKET-AF per } \\
\text { protocollo } \\
\text { HR (IC 95\%) }\end{array}$ & $\begin{array}{l}\text { ARISTOTLE } \\
\text { HR (IC 95\%) }\end{array}$ \\
\hline Ictus ischemico & $1,11(0,88-1,39)$ & $0,76(0,59-0,97)^{*}$ & $0,94(0,75-1,17)$ & $0,92(0,74-1,13)$ \\
\hline $\begin{array}{l}\text { Endpoint combinato ictus ischemico, } \\
\text { emorragico, embolismo sistemico }\end{array}$ & $0,90(0,74-1,10)$ & $0,66(0,52-0,81)^{*}$ & $0,79(0,66-0,96)^{*}$ & $0,79(0,66-0,95)^{*}$ \\
\hline Ictus disabilitante o fatale & $0,93(0,72-1,21)$ & $0,66(0,50-0,87)^{*}$ & NR & $0,71(0,54-0,94)^{*}$ \\
\hline Mortalità per cause vascolari & $0,90(0,77-1,06)$ & $0,85(0,72-0,99)^{*}$ & $0,89(0,73-1,10)$ & $0,89(0,76-1,04)$ \\
\hline Mortalità totale & $0,91(0,80-1,03)$ & $0,88(0,77-1,00)$ & $0,85(0,70-1,02)$ & $0,89(0,80-0,98)^{*}$ \\
\hline Emorragie totali & $0,78(0,73-0,83)^{*}$ & $0,91(0,85-0,96)^{*}$ & $1,03(0,96-1,11)$ & $0,71(0,68-0,75)^{*}$ \\
\hline Emorragie maggiori & $0,80(0,70-0,93)^{*}$ & $0,93(0,81-1,07)$ & $1,04(0,90-1,20)$ & $0,69(0,60-0,80)^{*}$ \\
\hline Emorragie fatali & $0,58(0,35-0,97)^{*}$ & $0,70(0,43-1,14)$ & $0,50(0,31-0,79)$ & $\begin{array}{c}\text { NR (rischio apixaban } \\
0,0037 \text { vs rischio } \\
\text { warfarin } 0,0060 \text { ) }\end{array}$ \\
\hline Emorragie intracraniche & $0,30(0,19-0,45)^{*}$ & $0,41(0,28-0,60)^{*}$ & $0,67(0,47-0,93)^{*}$ & $0,42(0,30-0,58)^{*}$ \\
\hline Emorragie gastrointestinali & $1,08(0,85-1,38)$ & $1,48(1,18-1,85)^{*}$ & $3,2 \%$ vs $2,2 \%, p<0,001$ & $0,89(0,70-1,15)$ \\
\hline $\begin{array}{l}\text { Beneficio netto: eventi vascolari, } \\
\text { emorragie maggiori, mortalità }\end{array}$ & $0,92(0,84-1,01)$ & $0,90(0,82-0,99)^{*}, \S$ & NR & $0,85(0,78-0,92)^{*}$ \\
\hline
\end{tabular}


Dabigatran 150 mg bis/die è risultato superiore al warfarin nel ridurre anche gli stroke ischemici come endpoint singolo.

Seppur dimostrando una riduzione del rischio relativo, tuttavia, i nuovi anticoagulanti orali non sembrano ridurre in maniera significativa la mortalità per tutte le cause rispetto ai farmaci VKA, con l'eccezione di apixaban che raggiunge questo obiettivo con una differenza ai limiti della significatività statistica; dabigatran a entrambi i dosaggi e rivaroxaban risultano non inferiori al warfarin su questo endpoint [39-42].

I risultati di questi RCT sembrano aprire la strada all'utilizzo dei nuovi anticoagulanti orali nella profilassi antitromboembolica del paziente con FA non valvolare anche in termini di prima scelta in tale ambito al pari dei farmaci VKA. Al momento, solo per dabigatran ciò è già stato recepito in un aggiornamento dalle linee guida americane, in cui il farmaco è raccomandato con grado IB (raccomandazione forte alla somministrazione derivante da un singolo trial) come alternativa al warfarin in pazienti con FA non valvolare emodinamicamente stabili con clearance della creatinina $>15 \mathrm{~mL} /$ min e senza gravi alterazioni epatiche [43].

I nuovi anticoagulanti orali sembrano essere, oltre che non inferiori ai farmaci VKA, superiori all'ASA in questo contesto. Un recente RCT di fase III, AVERROES (Apixaban versus ASA to Prevent Stroke in Atrial Fibrillation Patients Who Have Failed or Are Unsuitable for Vitamin K Antagonist Treatment), ha confrontato uno dei tre nuovi anticoagulanti, l'apixaban, con ASA nella profilassi antitrombotica dei pazienti con FA che non potevano essere trattati con farmaci VKA per controindicazioni assolute o relative [44]. Questo studio ha dimostrato che apixaban riduce del $55 \%$ il rischio relativo di tromboembolismo cerebrale e sistemico e del $18 \%$ il rischio relativo di morte per tutte le cause rispetto all'ASA al dosaggio di 81-324 mg, senza aumentare il rischio di emorragia maggiore $(1,4$ per 100 pazienti/anno nel gruppo apixaban vs 1,2 nel gruppo ASA, $\mathrm{p}=$ non significativa) e intracranica $(0,4$ per 100 pazienti $/$ anno in entrambi i gruppi, $p=$ non significativa) [44].

È quindi verosimile, alla luce di questi risultati, l'inizio di una nuova era nella profilassi antitrombotica del paziente con FA non valvolare che vedrà protagonisti i nuovi farmaci anticoagulanti orali.

\section{Problematiche aperte}

Nonostante i promettenti risultati derivanti dagli RCT sopra descritti, è tuttavia importante sottolineare che molti aspetti restano da chiarire e molte domande non hanno ancora trovato risposte definitive. Nel seguito se ne espongono alcune.

\section{Come confrontare tra loro i nuovi farmaci anticoagulanti orali e quale scegliere nella pratica clinica?}

Lo scopo della presente revisione è fornire un'analisi dei risultati degli RCT relativi alla profilassi antitromboembolica dei pazienti con FA non valvolare con nuovi anticoagulanti orali confrontati con il warfarin. Fermo restando che nella pratica clinica ogni clinico valuterà la scelta di un nuovo anticoagulante orale sulla base delle proprie conoscenze ed esperienze e dei risultati derivanti dalla diffusa applicazione nella vita reale dei nuovi farmaci, al momento non sembra possibile fornire indicazioni sulla superiorità di un farmaco rispetto a un altro alla luce degli RCT di fase III. In effetti, benché i disegni degli studi siano in parte simili, alcune caratteristiche delle popolazioni in oggetto appaiono differenti e pertanto i dati di efficacia e sicurezza devono essere letti con molta cautela quando l'obiettivo è quello di paragonare un nuovo anticoagulante con un altro. Se da un lato, infatti, nello studio RE-LY la stessa molecola a dosaggi differenti ha ottenuto risultati diversi di efficacia (il dosaggio di $150 \mathrm{mg} 2$ volte/die è superiore in termini di riduzione del rischio embolico rispetto al warfarin e al dosaggio di $110 \mathrm{mg} 2$ volte/die) e di sicurezza (il dosaggio di $150 \mathrm{mg} 2$ volte/die si associa a un più alto rischio emorragico rispetto al dosaggio $110 \mathrm{mg} 2$ volte/die, ma non rispetto al warfarin di cui è superiore a eccezione del rischio emorragico gastrointestinale) $[39,40]$, dall'altro lato sarebbe azzardato estrapolare come dato assoluto il maggior rischio emorragico, rispetto alle altre nuove molecole anticoagulanti, di rivaroxaban, che peraltro ha un ottimo profilo di efficacia (RRR di eventi embolici pari al 20,5\%, inferiore solo a quello di dabigatran $150 \mathrm{mg}$ bis/die) [41].

Come già sottolineato, la popolazione inclusa nel ROCKET$\mathrm{AF}$ ha caratteristiche intrinseche che potrebbero elevare il rischio emorragico, dal momento che l' $87 \%$ di essa ha un punteggio $\mathrm{CHADS}_{2} \geq 3$ contro poco più del $30 \%$ degli altri due RCT [41]. Una recente analisi post hoc del RE-LY potrebbe altresì confermare questa ipotesi, dal momento che essa evidenzia come il rischio embolico, emorragico e di mortalità, sia vascolare che per tutte le cause, aumenti in maniera esponenziale all'aumentare del punteggio $\mathrm{CHADS}_{2}$, pur mantenendosi l'obiettivo di non inferiorità di dabigatran a entrambi i dosaggi su warfarin per gli endpoint sopra considerati [45]. In particolare, nei pazienti con score $\mathrm{CHADS}_{2} \geq 2$ entrambi i dosaggi di dabigatran perderebbero gran parte del loro beneficio in termini di emorragie maggiori, con una RRR del dosaggio $110 \mathrm{mg}$ bis/die che passa dal $20 \%$ al $12 \%$, e al dosaggio di $150 \mathrm{mg}$ bis/die si ha addirittura un vantaggio del warfarin, con una RRR del farmaco VKA che va da $-7 \% a+46 \%$. In questi pazienti (score $\mathrm{CHADS}_{2} \geq 2$ ) non si perde comunque il vantaggio nella RRR di emorragie intracraniche, che rimane sostanzialmente invariata (72\% vs 70\%) al dosaggio $110 \mathrm{mg}$ bis/die, ma si riduce al $17,8 \%$ vs il $59 \%$ emerso nel dato complessivo (score $\mathrm{CHADS}_{2}$ 0-6) per il dosaggio $150 \mathrm{mg}$ bis/die [45]. Non sono disponibili dati che forniscano, nei tre RCT, il rischio emorragico dei pazienti mediante score, quali l'HAS-BLED o altri.

Occorre altresì sottolineare che la scelta del singolo nuovo anticoagulante dovrebbe essere ponderata anche sulla base delle caratteristiche farmacodinamiche e farmacocinetiche dello stesso. $\grave{E}$ evidente che nei pazienti con insufficienza renale moderata (clearance della creatinina $30-50 \mathrm{~mL}$ ) potrebbero essere privilegiati rivaroxaban o apixaban, che sono escreti in forma attiva per via renale solo per un terzo, mentre il dabigatran è eliminato quasi interamente per via renale. È comunque opportuno sottolineare che nello studio RE-LY non sono stati esclusi pazienti con tale ridotta funzionalità renale. In ogni caso in questa condizione di ridotta funzionalità renale si dovrebbero considerare dosi ridotte dei nuovi farmaci e andrebbero attentamente monitorati i valori di creatinina, specie nei pazienti in cui se ne preveda il declino. È degno di nota che, nel ROCKET-AF, l'aggiustamento posologico nei pazienti con insufficienza renale moderata (15 mg anziché $20 \mathrm{mg}$ ) - popolazione a rischio aumentato di eventi sia embolici sia emorragici - ha comportato comunque il raggiungimento della non inferiorità rispetto 
al warfarin per quanto riguarda sia l'efficacia sia la sicurezza [46]. Per il dabigatran vengono consigliate riduzioni del dosaggio nel paziente ultraottantenne.

In ultima analisi la risposta al quesito iniziale dovrà provenire da studi di confronto tra i nuovi anticoagulanti orali. Al momento attuale gli autori del presente articolo ritengono che non sia possibile fornire una risposta obiettiva al quesito posto sulla scorta degli studi RE-LY, ROCKET-AF e ARISTOTLE.

\section{La non inferiorità dei nuovi anticoagulanti orali persiste nei pazienti in terapia con farmaci VKA adeguatamente condotta?}

Un'analisi dello studio RE-LY sembra dimostrare che, dopo suddivisione dei pazienti in quartili in base al TTR $(<57,1 \%$, $57,1-65,4 \%, 65,5-72,6 \%,>72,6 \%$ ), il beneficio netto di dabigatran nei confronti di warfarin potrebbe ridursi, rimanendo comunque sempre a favore di dabigatran nei pazienti con controllo ottimale dell'INR rappresentato da un TTR $>72,6 \%$ [47], fermi restando i benefici pratici (controllo dell'INR, interazioni con la dieta, ecc. Nel ROCKET-AF la non inferiorità di rivaroxaban su warfarin si manterrebbe anche nel quartile TTR 65,7-100\% (HR 0,77, IC 95\% 0,49-1,12) [48]. Rimangono in ogni modo da stabilire la reale fattibilità di un tale livello di aderenza alla terapia nella pratica clinica e il reale beneficio in termini di farmacoeconomia.

\section{Com'è controllabile l'aderenza alla terapia con i nuovi farmaci anticoagulanti orali? Non necessitano realmente di un controllo di laboratorio?}

Una delle principali difficoltà potenzialmente riscontrabili nella futura utilizzazione dei nuovi farmaci anticoagulanti orali attiene all'aderenza alla terapia, facilmente valutabile con i farmaci VKA mediante il dosaggio dell'INR. I nuovi farmaci anticoagulanti orali hanno, tra le loro caratteristiche farmacocinetiche e farmacodinamiche, la proprietà di presentare un profilo dose-risposta costante e, pertanto, sono stati studiati clinicamente in assenza di un controllo specifico di laboratorio. Il tempo di trombina su plasma diluito e il tempo di coagulazione da ecarina sembrano essere i test migliori per valutare l'attività anticoagulante del dabigatran, ma al momento sono di difficile applicazione nella pratica quotidiana. Il tempo di tromboplastina parziale attivato (aPTT) è un test ampiamente disponibile e può offrire un'approssimativa indicazione dell'intensità dell'anticoagulazione raggiunta con dabigatran, specie nei pazienti con sanguinamento in atto o ad alto rischio di sanguinamento; va ribadito che la sensibilità dell'aPTT è molto limitata e, quindi, il test non garantisce una precisa quantificazione dell'effetto anticoagulante di dabigatran, specie in caso di eccessivi livelli plasmatici del farmaco. I test per l'attività anti-Xa e, limitatamente al rivaroxaban, il tempo di protrombina (PT) sembrano invece essere i metodi più adatti per valutare l'attività anticoagulante degli inibitori del fattore Xa $[33,38,49,50]$. L'applicazione di test di laboratorio per il monitoraggio dell'effetto anticoagulante dei nuovi farmaci potrebbe essere considerata in alcune particolari situazioni cliniche già menzionate come, per esempio, in caso di eventi emorragici o nelle situazioni in cui sia necessario sapere con precisione se il paziente è aderente alla terapia (eventi embolici, necessità di interventi chirurgici o necessità di sospendere la terapia, ecc). Sono ancora allo studio metodiche di laboratorio, più sensibili e se possibile specifiche, finalizzate a questo obiettivo [51-54]. È quindi fondamentale educare i pazienti all'assunzione dei nuovi farmaci in maniera scrupolosa e costante, così com'è fondamentale un periodico followup clinico di questi pazienti.

Che cosa fare nel caso di un paziente con nuova evidenza di FA e non ancora in trattamento con farmaci VKA? E nei pazienti già in trattamento con farmaci VKA è consigliabile la loro prosecuzione o l'eventuale passaggio ai nuovi farmaci?

Le risposte a tali quesiti sono particolarmente difficili e rappresenteranno una sfida per il clinico. Nei pazienti con FA di nuova diagnosi e non precedentemente trattati con VKA, è suggerito il trattamento come prima scelta con i nuovi farmaci anticoagulanti orali soprattutto nei soggetti ad alto rischio tromboembolico ed emorragico $\left(\mathrm{CHA}_{2} \mathrm{DS}_{2}-\mathrm{VASC} \geq 3 \mathrm{e}\right.$ HAS-BLED > 3). Le comorbilità, i concomitanti trattamenti con altri farmaci potenzialmente interferenti con enzimi implicati nel metabolismo del warfarin, potrebbero influenzare sia la decisione di intraprendere/non intraprendere il trattamento con nuovi farmaci anticoagulanti orali, sia la scelta del singolo nuovo anticoagulante orale. Sarà pertanto da ponderare con attenzione il dabigatran nei pazienti con insufficienza renale moderato-severa, mentre in tale situazione rivaroxaban e apixaban potrebbero rappresentare, come già sottolineato, la prima scelta date le loro proprietà farmacodinamiche $[55,56]$. Nel paziente che lamenta dispepsia o che ha problematiche di cardiopatia ischemica dabigatran potrebbe trovare minore indicazione, così come nei pazienti ad alto rischio di sanguinamento gastrointestinale dabigatran e rivaroxaban dovrebbero essere ben ponderati. L'associazione dei nuovi anticoagulanti orali con altri farmaci antitrombotici, come gli antiaggreganti piastrinici, potrebbe determinare un incremento degli eventi emorragici - come dimostrato dagli studi APPRAISE (Apixaban for Prevention of Acute Ischemic and Safety Events) I e II con apixaban nel paziente con sindrome coronarica - e dovrebbe, quindi, essere attentamente valutata [57,58].

Milioni di persone sono attualmente in terapia con $i$ farmaci VKA; esse potranno disporre di un'alternativa farmacologica altrettanto efficace e sicura, ma la scelta di passare/non passare alla nuova terapia dovrà essere personalizzata sul singolo caso. I pazienti con scarsa aderenza alla proposta terapeutica, con labilità dei valori di INR o scarso controllo qualitativo della terapia con i farmaci VKA, oppure coloro che hanno avuto eventi tromboembolici o emorragici nel corso dell'assunzione di farmaci VKA o hanno un alto rischio trombotico, potranno essere candidati alla terapia con i nuovi anticoagulanti orali [55,56]. Altre categorie di pazienti potenzialmente da convertire ai nuovi anticoagulanti orali potrebbero essere quelli in cui il disagio o le difficoltà individuali o logistiche nel monitoraggio laboratoristico dell'INR siano chiaramente evidenti (pazienti giovani, professionisti, pazienti isolati al domicilio o per $i$ quali esistono problemi logistici nella pianificazione del monitoraggio, ecc.) o coloro per i quali le interazioni farmacologiche e/o dietetiche potrebbero influire negativamente sulla qualità di vita e sull'efficacia/sicurezza terapeutica dei farmaci VKA $[55,56]$. I pazienti in terapia con VKA con un controllo qualitativo ottimale (TTR $>75 \%$ ), soprattutto se a basso rischio trombotico $\left(\mathrm{CHA}_{2} \mathrm{DS}_{2}-\mathrm{VASC} \leq 2\right)$ ed emorragico 
(HAS-BLED $<3$ ), potranno essere indirizzati alla prosecuzione della stessa. Occorre altresì richiamare l'attenzione sul fatto che la gran parte degli eventi emorragici che avvengono in corso di terapia con farmaci VKA, si verifica in pazienti all'interno dell'intervallo terapeutico e d'altro canto, come già evidenziato, una quota non trascurabile di pazienti che assumono VKA è colpita da eventi embolici pur in presenza di un adeguato INR $[29,30,59,60]$. In definitiva, risulta oltremodo difficile prendere una posizione al fine della riduzione degli eventi embolici o emorragici basandosi sulla qualità del controllo della terapia con VKA.

\section{Qual è il rapporto costo/beneficio dei nuovi anticoagulanti orali?}

Le differenze geografiche e di organizzazione dei vari Sistemi Sanitari Regionali determinano una discrepanza nella valutazione del rapporto rischio/beneficio. Se è ovvio che il costo del farmaco è sicuramente superiore per i nuovi anticoagulanti orali, al momento, risulta difficile soprattutto la stima dei costi indiretti, ascrivibili primariamente al costo del monitoraggio laboratoristico comprensivo della componente medica, della componente strumentale vera e propria e dei costi relativi alla riduzione/gestione degli eventi embolici/ emorragici. Gli studi clinici di farmacoeconomia a oggi pubblicati sembrerebbero comunque indirizzare verso un favorevole rapporto costo/beneficio per i nuovi anticoagulanti orali [61-63], specialmente nei pazienti ad alto rischio e in coloro per i quali la qualità del controllo dell'INR risulti scarsa (TTR $<57 \%$ ) [64].

\section{Come comportarsi per la terapia di ricoagulazione urgente "reversal therapy"?}

Una delle principali, possibili limitazioni all'uso dei nuovi anticoagulanti orali potrebbe essere costituita dalla mancanza di antidoti specifici in presenza di un evento emorragico, specie se maggiore o a rischio di vita, in pazienti che assumono tali farmaci. È ben nota, seppur assolutamente sottoutilizzata, la possibilità di effettuare efficacemente e prontamente una terapia di ricoagulazione urgente dei farmaci VKA mediante l'infusione endovenosa di concentrato di complesso protrombinico (CCP), plasma fresco congelato (PFC) e vitamina K1 [65,66]. A oggi non è ancora possibile disporre di un antidoto specifico per la terapia di ricoagulazione urgente dei nuovi anticoagulanti orali [67]. Studi sperimentali condotti su animali dimostrano che il pretrattamento con i nuovi anticoagulanti orali non si associa a espansione significativa dell'emorragia cerebrale provocata [68], a differenza di quanto avviene con i farmaci VKA condizionandone la prognosi negativa [69]. Tale aspetto deve essere valutato e approfondito nella pratica reale, dal momento che non sono ancora stati pubblicati studi relativi alle caratteristiche cliniche delle emorragie intracraniche né maggiori che interessano altri organi critici (peritoneo, retroperitoneo, torace, apparato gastroenterico ecc.).

Vale la pena sottolineare come, anche nelle emorragie intracraniche associate a farmaci antiaggreganti piastrinici, siano privilegiate solo le misure di supporto, medico-conservative o chirurgiche, ignorando potenziali strategie terapeutiche (la trasfusione di concentrati piastrinici e/o l'infusione di desmopressina) attualmente disponibili seppur con un basso grado di raccomandazione [70,71]. Studi recenti che hanno valutato l'effetto dei CCP nella ricoagulazione urgente dei soggetti trattati con i nuovi anticoagulanti orali hanno portato a risultati discordanti. Se da un lato, in volontari sani, la ricoagulazione mediante CCP durante terapia con rivaroxaban è testimoniata dalla normalizzazione dei parametri coagulativi [72], dall'altro lato il CCP non sembra avere effetto emostatico in ratti pretrattati con rivaroxaban e sottoposti a sanguinamento splancnico [73]. Occorre peraltro sottolineare che il CCP sembra non determinare la normalizzazione dei parametri coagulativi in volontari sani trattati con dabigatran [72]. Pertanto si è ancora lontani dal disporre di uno o più antidoti specifici per i nuovi anticoagulanti orali, particolarmente indicati nelle emorragie maggiori o a rischio di vita. In attesa di ulteriori sperimentazioni cliniche, a oggi vengono consigliati per la ricoagulazione urgente dei farmaci inibitori diretti della trombina, PFC, fattore VII ricombinante attivato (FVIIra), i CCP attivati (cosiddetti FEIBA) utilizzati nel paziente emofilico che sviluppa inibitori del fattore VIII, oppure, in ultima analisi, tecniche emodialitiche [72,73]. Per la ricoagulazione urgente degli inibitori del fattore $\mathrm{Xa}$ sono consigliati CCP, FEIBA, PFC o FVIIra [74,75]. Nelle emorragie minori, la sospensione del farmaco, potrebbe essere sufficiente data la breve emivita dei nuovi anticoagulanti orali $[74,75]$.

\section{Conclusioni}

Gli studi RE-LY, ROCKET-AF e ARISTOTLE dimostrano che $i$ nuovi anticoagulanti orali possono costituire un'alternativa efficace e sicura al warfarin nella profilassi tromboembolica del paziente con FA non valvolare. La prossima diffusione di questi farmaci permetterà di confermarne la validità nella pratica clinica reale e di fornire risposta alle diverse problematiche non ancora risolte.

\section{Nota aggiuntiva}

Gli autori precisano che dopo l'invio e l'accettazione del presente manoscritto sono state pubblicate le nuove linee guida ACCP IX Edizione 2012 [76]. Queste raccomandano di effettuare la stima del rischio tromboembolico mediante lo score $\mathrm{CHADS}_{2}$. Le nuove linee guida ACCP IX Edizione raccomandano dabigatran o VKA con INR target 2.5 nel paziente con FA ad alto rischio tromboembolico $\left(\mathrm{CHADS}_{2} \geq 2\right.$ ) (grado IA). In questa tipologia di pazienti le nuove linee guida suggeriscono di preferire dabigatran $150 \mathrm{mg}$ bis/die ai VKA (grado IIB). Nei pazienti ad alto rischio tromboembolico che non sono suscettibili alla o che rifiutano la terapia con anticoagulante orale con dabigatran o VKA, le linee guida ACCP IX Edizione raccomandano di preferire l'associazione ASAclopidogrel piuttosto che ASA da solo (grado IB) [76]. Nel paziente a rischio tromboembolico intermedio $\left(\mathrm{CHADS}_{2}=1\right)$ viene suggerito di preferire terapia anticoagulante orale con dabigatran $150 \mathrm{mg} / \mathrm{bis} /$ die o VKA con INR target 2.5 rispetto a nessuna terapia (grado IB) e rispetto all'associazione ASAclopidogrel od ASA 75-325 mg da solo (grado IIB). Nei pazienti a rischio intermedio che non sono suscettibili alla o che rifiutano la terapia con anticoagulante orale con dabigatran o VKA, viene raccomandato di preferire l'associazione ASAclopidogrel piuttosto che ASA 75-325 mg da solo (grado IIB). Infine nel paziente a basso rischio tromboembolico $\left(\mathrm{CHADS}_{2}=0\right)$ 
le nuove linee guida ACCP raccomandano nessuna terapia antitrombotica o ASA al dosaggio 75-325 mg (grado IIB) [76].

Deve essere peraltro sottolineato che gli Autori delle nuove linee guida ACCP 2012, al momento della loro stesura, non hanno preso in considerazione rivaroxaban ed apixan come potenziali opzioni terapeutiche perchè ancora non autorizzati dagli Enti regolatori (Food and Drug Administration, FDA) per questa indicazione [76].

\section{Conflitto di interessi}

Luca Masotti, Mario Di Napoli, Davide Imberti, Daniel Godoy, Grazia Panigada, Niccolò Napoli, Giancarlo Landini, Roberto Cappelli dichiarano di non avere nessun conflitto di interessi.

Walter Ageno dichiara di aver partecipato a advisory board organizzati da Bayer Schering Pharma e BMS/Pfizer e di aver ricevuto compensi per relazioni a congressi da Bayer Schering Pharma, BMS/Pfizer e Boheringer Ingelheim.

Ido lori dichiara di aver ricevuto compensi per advisory board da Boheringer Ingelheim Italia e da Pfizer.

Domenico Prisco dichiara di aver ricevuto compensi per letture e advisory board da GSK, Bayer Schering Pharma e Daiichi Sankyo.

Giancarlo Agnelli dichiara di aver ricevuto compensi per letture e advisory board da Bayer Schering Pharma, BMS/ Pfizer e Boheringer Ingelheim.

\section{Allegato 1. $\mathrm{CHADS}_{2}$ e $\mathrm{CHA}_{2} \mathrm{DS}_{2}$-VASC}

\begin{tabular}{clc}
\hline & \multicolumn{1}{c}{ CHADS $_{2}$} \\
\hline Lettera dell'acronimo & \multicolumn{1}{c}{ Variabile } & Punteggio \\
\hline C & Congestive heart failure & 1 punto \\
\hline H & Hypertension & 1 punto \\
\hline A & Age $>75$ years & 1 punto \\
\hline D & Diabetes & 1 punto \\
\hline$S_{2}$ & Prior Stroke/TIA & 2 punti \\
\hline
\end{tabular}

Rischio annuale di ictus/embolismo sistemico

\begin{tabular}{ll}
\hline 0 & $1,9 \%$ \\
\hline 1 & $2,8 \%$ \\
\hline 2 & $4,0 \%$ \\
\hline 3 & $5,9 \%$ \\
\hline 4 & $8,5 \%$ \\
\hline 5 & $12,5 \%$ \\
\hline 6 & $18,2 \%$ \\
\hline
\end{tabular}

$\mathrm{CHA}_{2} \mathrm{DS}_{2}$-VASC

\begin{tabular}{lll}
\hline C & Congestive heart failure & 1 punto \\
\hline $\mathrm{H}$ & Hypertension & 1 punto \\
\hline $\mathrm{A}_{2}$ & Age $>75$ years & 2 punti \\
\hline $\mathrm{D}$ & Diabetes & 1 punto \\
\hline $\mathrm{S}_{2}$ & Prior Stroke/TIA & 2 punti \\
\hline
\end{tabular}

\begin{tabular}{|c|c|c|}
\hline $\mathrm{V}$ & Vascular diseases & 1 punto \\
\hline A & Age $65-74$ anni & 1 punto \\
\hline $\mathrm{SC}$ & Sex Category (female) & 1 punto \\
\hline \multicolumn{3}{|c|}{ Rischio annuale di ictus/embolismo sistemico } \\
\hline 0 & $0 \%$ & \\
\hline 1 & $1,3 \%$ & \\
\hline 2 & $2,2 \%$ & \\
\hline 3 & $3,2 \%$ & \\
\hline 4 & $4,0 \%$ & \\
\hline 5 & $6,7 \%$ & \\
\hline 6 & $9,8 \%$ & \\
\hline 7 & $9,6 \%$ & \\
\hline 8 & $6,7 \%$ & \\
\hline 9 & $15,2 \%$ & \\
\hline
\end{tabular}

\section{Allegato 2. Stima del rischio emorragico: score HAS-BLED e ATRIA}

HAS-BLED

Un punteggio $\geq 3$ identifica pazienti con un rischio di emorragia a 12 mesi $>3,5 \%$

\begin{tabular}{clc}
\hline $\begin{array}{c}\text { Lettera } \\
\text { dell'acronimo }\end{array}$ & \multicolumn{1}{c}{ Variabile } & Punteggio \\
\hline $\mathrm{H}$ & $\begin{array}{l}\text { Hypertension } \\
\text { (ipertensione } \\
\text { arteriosa) }\end{array}$ & 1 \\
\hline $\mathrm{A}$ & $\begin{array}{l}\text { Abnormal renal } \\
\text { and liver function } \\
\text { (anormale funzione } \\
\text { epatica e renale) }\end{array}$ & 1 o 2 \\
\hline $\mathrm{S}$ & $\begin{array}{l}\text { Stroke (ictus) } \\
\mathrm{B}\end{array}$ & $\begin{array}{l}\text { Bleeding } \\
\text { (sanguinamento } \\
\text { precedente) }\end{array}$ \\
\hline $\mathrm{L}$ & $\begin{array}{l}\text { Labile INRs } \\
\text { (INR labile) }\end{array}$ & 1 \\
\hline $\mathrm{E}$ & $\begin{array}{l}\text { Elderly } \\
\text { (età }>65 \text { anni) }\end{array}$ & 1 \\
\hline $\mathrm{D}$ & $\begin{array}{l}\text { Drugs and } \\
\text { alchool } \\
\text { (farmaci e alcol) }\end{array}$ & 102 \\
\hline
\end{tabular}

ATRIA (Anticoagulation and Risk Factors in Atrial Fibrillation)

\begin{tabular}{lc}
\hline \multicolumn{1}{c}{ Variabile } & Punteggio \\
\hline Anemia & 3 \\
\hline Insufficienza renale severa & 3 \\
\hline
\end{tabular}




\begin{tabular}{|c|c|c|}
\hline \multicolumn{2}{|c|}{ Età $>75$ anni } & 2 \\
\hline Prece & dinamento & 1 \\
\hline Ipert & riosa & 1 \\
\hline Cla & del rischio & $\begin{array}{l}\text { Rischio di eventi } \\
\text { emorragici per anno } \\
\text { di trattamento }\end{array}$ \\
\hline $0-3$ & Basso & $0,8 \%$ \\
\hline 4 & Intermedio & $2,6 \%$ \\
\hline $5-10$ & Alto & $5,8 \%$ \\
\hline
\end{tabular}

\section{Allegato 3. Proprietà farmacocinetiche e farmacodinamiche dei nuovi anticoagulanti orali}

\begin{tabular}{|c|c|c|c|}
\hline & Dabigatran & Rivaroxaban & Apixaban \\
\hline Profarmaco & Dabigatran etexilato & No & No \\
\hline Peso molecolare & $\begin{array}{c}628 \mathrm{kD} \text { profarmaco (etexilato) } \\
471 \mathrm{kD} \text { farmaco }\end{array}$ & $436 \mathrm{kD}$ & $460 \mathrm{kD}$ \\
\hline Emivita plasmatica & $\begin{array}{l}\text { 7-9 ore dopo la prima dose } \\
12-14 \text { ore dopo multiple dosi }\end{array}$ & $\begin{array}{l}9 \text { ore in giovani-adulti } \\
12 \text { ore in anziani }>75 \text { anni }\end{array}$ & 12 ore \\
\hline $\begin{array}{l}\text { Tempo impiegato per } \\
\text { raggiungere la } \\
\text { concentrazione } \\
\text { massima }\left(C_{\max }\right)\end{array}$ & $0,5-2$ ore & $2-4$ ore & 3 ore \\
\hline Biodisponibilità & $6,5 \%$ & $>80 \%$ & $>50 \%$ \\
\hline Via di eliminazione & Renale $85 \%$ & $\begin{array}{l}\text { Renale } 66 \% \text {, di cui il } \\
33 \% \text { immodificato } \\
\text { Bilio-fecale } 35 \%\end{array}$ & $\begin{array}{l}\text { Renale } 25 \% \\
\text { Fecale } 75 \%\end{array}$ \\
\hline Legame con proteine plasmatiche & $35 \%$ & $90 \%$ & $85 \%$ \\
\hline Volume di distribuzione & $60-70 \mathrm{~L}$ & $0,6-1,5 \mathrm{~L} / \mathrm{kg}$ & $0,3 \mathrm{~L} / \mathrm{kg}$ \\
\hline Metabolismo del citocromo P450 & No & Sì & Sì \\
\hline Interazione con il cibo & No & No & No \\
\hline Interazione con farmaci & $\begin{array}{c}\text { Inibitori e induttori } \\
\text { di glicoproteina } \mathrm{P}, \\
\text { amiodarone, verapamil, } \\
\text { chinidina, } \\
\text { claritromicina }\end{array}$ & $\begin{array}{l}\text { Inibitori o induttori di } \\
\text { CYP3A4 e glicoproteina } \\
\text { P (ketokonazolo, } \\
\text { itraconazolo, ritonavir, } \\
\text { rifampicina, fenitoina, } \\
\text { carbamazepina) }\end{array}$ & $\begin{array}{c}\text { Inibitori o induttori } \\
\text { di CYP3A4 e glicoproteina P } \\
\text { (ketokonazolo, itraconazolo, } \\
\text { ritonavir, rifampicina, fenitoina, } \\
\text { carbamazepina) }\end{array}$ \\
\hline Frequenza di somministrazione & 2 volte/die & $1 \mathrm{volta} / \mathrm{die}$ & 2 volte/die \\
\hline Sicurezza in gravidanza & No & No & No \\
\hline Antidoto & $\begin{array}{c}\text { PFC? FEIBA? FVIIIra? } \\
\text { Dialisi? }\end{array}$ & CCP? FEIBA? PFC? FVIIra? & CCP? FEIBA? PFC? FVIIra? \\
\hline
\end{tabular}

Legenda: PFC = plasma fresco congelato; FEIBA = complesso protrombinico attivato (misurato in unità Feiba); FVIIra = fattore VII ricombinante attivato; $\mathrm{CCP}=$ concentrato di complesso protrombinico.

\section{Bibliografia}

[1] Camm AJ, Kirchhof P, Lip GY, Schotten U, Savelieva I, Ernst S, et al., European Heart Rhythm Association; European Associa- tion for Cardio-Thoracic Surgery. Guidelines for the management of atrial fibrillation: the Task Force for the Management of Atrial Fibrillation of the European Society of Cardiology (ESC). Eur Heart J 2010;31(19):2369-429. 
[2] Fuster V, Rydén LE, Cannom DS, Crijns HJ, Curtis AB, Ellenbogen $\mathrm{KA}$, et al., American College of Cardiology Foundation/American Heart Association Task Force. 2011 ACCF/AHA/HRS focused updates incorporated into the ACC/AHA/ESC 2006 guidelines for the management of patients with atrial fibrillation: a report of the American College of Cardiology Foundation/American Heart Association Task Force on practice guidelines. Circulation 2011;123(10):e269-367.

[3] Cairns JA, Connolly S, McMurtry S, Stephenson M, Talajic M, CCS Atrial Fibrillation Guidelines Committee. Canadian Cardiovascular Society atrial fibrillation guidelines 2010: prevention of stroke and systemic thromboembolism in atrial fibrillation and flutter. Can J Cardiol 2011;27(1):74-90.

[4] Go AS, Hylek EM, Phillips KA, Chang Y, Henault LE, Selby JV, et al. Prevalence of diagnosed atrial fibrillation in adults: national implications for rhythm management and stroke prevention: the AnTicoagulation and Risk Factors in Atrial Fibrillation (ATRIA) Study. JAMA 2001;285(18):2370-5.

[5] Miyasaka Y, Barnes ME, Gersh BJ, Cha SS, Bailey KR, Abhayaratna WP, et al. Secular trends in incidence of atrial fibrillation in Olmsted County, Minnesota, 1980 to 2000 , and implications on the projections for future prevalence. Circulation 2006;114(2): 119-25.

[6] Heeringa J, van der Kuip DA, Hofman A, Kors JA, van Herpen G, Stricker $\mathrm{BH}$, et al. Prevalence, incidence and lifetime risk of atrial fibrillation: the Rotterdam study. Eur Heart J 2006;27(8): 949-53.

[7] Lloyd-Jones DM, Wang TJ, Leip EP, Larson MG, Levy D, Vasan RS, et al. Lifetime risk for development of atrial fibrillation: the Framingham Heart Study. Circulation 2004;110(9):1042-6.

[8] Campanini M, Frediani R, Artom A, Pinna G, Valerio A, La Regina $M$, et al., on behalf of the FALP Study Group. Real-world management of atrial fibrillation in Internal Medicine units: the FA'FALP' observational study. J Cardiovasc Med (Hagerstown) 2011.

[9] Stewart S, Hart CL, Hole DJ, McMurray JJ. A population-based study of the long-term risks associated with atrial fibrillation: 20-year follow-up of the Renfrew/Paisley study. Am J Med 2002;113(5):359-64.

[10] Wolf PA, Abbott RD, Kannel WB. Atrial fibrillation as an independent risk factor for stroke: the Framingham Study. Stroke 1991;22(8):983-8.

[11] Stroke Risk in Atrial Fibrillation Working Group. Independent predictors of stroke in patients with atrial fibrillation: a systematic review. Neurology 2007;69(6):546-54.

[12] Gage BF, Waterman AD, Shannon W, Boechler M, Rich MW, Radford MJ. Validation of clinical classification schemes for predicting stroke: results from the National Registry of Atrial Fibrillation. JAMA 2001;285(22):2864-70.

[13] Lin HJ, Wolf PA, Kelly-Hayes M, Beiser AS, Kase CS, Benjamin EJ, et al. Stroke severity in atrial fibrillation. The Framingham Study. Stroke 1996;27(10):1760-4.

[14] Steger C, Pratter A, Martinek-Bregel M, Avanzini M, Valentin A, Slany J, et al. Stroke patients with atrial fibrillation have a worse prognosis than patients without: data from the Austrian Stroke registry. Eur Heart J 2004;25(19):1734-40.

[15] Kolominsky-Rabas PL, Weber M, Gefeller O, Neundoerfer B, Heuschmann PU. Epidemiology of ischemic stroke subtypes according to TOAST criteria: incidence, recurrence, and longterm survival in ischemic stroke subtypes: a population-based study. Stroke 2001;32(12):2735-40.

[16] Hart RG, Pearce LA, Aguilar MI. Meta-analysis: antithrombotic therapy to prevent stroke in patients who have nonvalvular atrial fibrillation. Ann Intern Med 2007;146(12):857-67.

[17] Mant J, Hobbs FD, Fletcher K, Roalfe A, Fitzmaurice D, Lip GY, et al., BAFTA investigators; Midland Research Practices Network (MidReC). Warfarin versus aspirin for stroke prevention in an elderly community population with atrial fibrillation (the
Birmingham Atrial Fibrillation Treatment of the Aged Study, BAFTA): a randomised controlled trial. Lancet 2007; 370(9586):493-503.

[18] Rash A, Downes T, Portner R, Yeo WW, Morgan N, Channer KS. A randomised controlled trial of warfarin versus aspirin for stroke prevention in octogenarians with atrial fibrillation (WASPO). Age Ageing 2007;36(2):151-6.

[19] Morgan CL, McEwan P, Tukiendorf A, Robinson PA, Clemens A, Plumb JM. Warfarin treatment in patients with atrial fibrillation: observing outcomes associated with varying levels of INR control. Thromb Res 2009;124(1):37-41.

[20] Connolly S, Pogue J, Hart R, Pfeffer M, Hohnloser S, Chrolavicius $S$, et al., ACTIVE Writing Group of the ACTIVE Investigatore. Clopidogrel plus aspirin versus oral anticoagulation for atrial fibrillation in the Atrial fibrillation Clopidogrel Trial with Irbesartan for prevention of Vascular Events (ACTIVE W): a randomised controlled trial. Lancet 2006;367(9526):1903-12.

[21] Connolly SJ, Pogue J, Hart RG, Hohnloser SH, Pfeffer M, Chrolavicius $S$, et al., ACTIVE Investigators. Effect of clopidogrel added to aspirin in patients with atrial fibrillation. $\mathrm{N}$ Engl $\mathrm{J}$ Med 2009;360(20):2066-78.

[22] Connolly SJ, Pogue J, Eikelboom J, Flaker G, Commerford P, Franzosi MG, et al., ACTIVE W Investigators. Benefit of oral anticoagulant over antiplatelet therapy in atrial fibrillation depends on the quality of international normalized ratio control achieved by centers and countries as measured by time in therapeutic range. Circulation 2008;118(20):2029-37.

[23] Connolly SJ, Eikelboom JW, Ng J, Hirsh J, Yusuf S, Pogue J, et al., ACTIVE (Atrial Fibrillation Clopidogrel Trial with Irbesartan for Prevention of Vascular Events) Steering Committee and Investigators. Net clinical benefit of adding clopidogrel to aspirin therapy in patients with atrial fibrillation for whom vitamin $\mathrm{K}$ antagonists are unsuitable. Ann Intern Med 2011; 155(9):579-86.

[24] Singer DE, Albers GW, Dalen JE, Fang MC, Go AS, Halperin JL, et al., American College of Chest Physicians. Antithrombotic therapy in atrial fibrillation: American College of Chest Physicians Evidence-Based Clinical Practice Guidelines ( $8^{\text {th }}$ Edition). Chest 2008;133(6 Suppl):546-92.

[25] SPREAD. Ictus cerebrale: linee guida italiane di prevenzione e trattamento. Milano: Pubblicazioni Catel Division; 2010.

[26] Wann LS, Curtis AB, January CT, Ellenbogen KA, Lowe JE, Estes NA, 3rd. et al., 2011 Writing Group Members, Fuster V, Rydén LE, Cannom DS, Le Heuzey JY, Crijns HJ, Lowe JE, et al; 2006 Writing Committee Members, Jacobs AK, Anderson JL, Albert N, Hochman JS, Buller CE, Kushner FG, et al; ACCF/AHA Task Force Members. 2011 ACCF/AHA/HRS focused update on the management of patients with atrial fibrillation (updating the 2006 guideline): a report of the American College of Cardiology Foundation/American Heart Association Task Force on Practice Guidelines. Circulation 2011;123(1):104-23.

[27] Ogilvie IM, Newton N, Welner SA, Cowell W, Lip GY. Underuse of oral anticoagulants in atrial fibrillation: a systematic review. Am J Med 2010;123(7):638-45.

[28] Ferro D, Loffredo L, Polimeni L, Violi F. Underuse of oral anticoagulants in patients with nonvalvular atrial fibrillation in Italy. Intern Emerg Med 2007;2(1):24-8.

[29] Paciaroni M, Agnelli G, Caso V, Venti M, Milia P, Silvestrelli G, et al. Atrial fibrillation in patients with first-ever stroke: frequency, antithrombotic treatment before the event and effect on clinical outcome. J Thromb Haemost 2005;3(6):1218-23.

[30] Gandolfo C, Balestrino M, Burrone A, Del Sette M, Finocchi C. Stroke due to atrial fibrillation and the attitude to prescribing anticoagulant prevention in Italy. A prospective study of a consecutive stroke population admitted to a comprehensive stroke unit. J Neurol 2008;255(6):796-802.

[31] Levi M, Hobbs FD, Jacobson AK, Pisters R, Prisco D, Bernardo A, et al. Improving antithrombotic management in patients with 
atrial fibrillation: current status and perspectives. Semin Thromb Hemost 2009;35(6):527-42.

[32] Paciaroni M, Agnelli G, Ageno W, Caso V, Corea F, Lanari A, et al. Risk factors for cerebral ischemic events in patients with atrial fibrillation on warfarin for stroke prevention. Atherosclerosis 2010;212(2):564-6.

[33] Ansell J, Hirsh J, Hylek E, Jacobson A, Crowther M, Palareti G. American College of Chest Physicians. Pharmacology and management of the vitamin $\mathrm{K}$ antagonists: American College of Chest Physicians Evidence-Based Clinical Practice Guidelines ( $8^{\text {th }}$ Edition). Chest 2008;133(6 Suppl):160-98.

[34] Schulman S, Beyth RJ, Kearon C, Levine MN, American College of Chest Physicians. Hemorrhagic complications of anticoagulant and thrombolytic treatment: American College of Chest Physicians Evidence-Based Clinical Practice Guidelines ( $8^{\text {th }}$ Edition). Chest 2008;133(6 Suppl):257-98

[35] Wittkowsky AK. Effective anticoagulation therapy: defining the gap between clinical studies and clinical practice. Am J Manag Care 2004;10(10 Suppl):S297-306.

[36] Masotti L, Becattini C, Cappelli R, Landini G, Pampana A, Prisco $D$, et al. Efficacy and safety of new oral anticoagulants in prophylaxis and treatment of venous thromboembolism. Rev Health Care 2010 7-26.

[37] Fang MC, Go AS, Chang Y, Borowsky LH, Pomernacki NK, Udaltsova $\mathrm{N}$, et al. A new risk scheme to predict warfarin-associated hemorrhage: The ATRIA (Anticoagulation and Risk Factors in Atrial Fibrillation) Study. J Am Coll Cardiol 2011;58(4):395-401.

[38] Weitz JI, Hirsh J, Samama MM, American College of Chest Physicians. New antithrombotic drugs: American College of Chest Physicians Evidence-Based Clinical Practice Guidelines ( $8^{\text {th }}$ Edition). Chest 2008;133(6 Suppl):234-56.

[39] Connolly SJ, Ezekowitz MD, Yusuf S, Eikelboom J, Oldgren J, Parekh A, et al., RE-LY Steering Committee and Investigators. Dabigatran versus warfarin in patients with atrial fibrillation. $\mathrm{N}$ Engl J Med 2009;361(12):1139-51.

[40] Connolly SJ, Ezekowitz MD, Yusuf S, Reilly PA, Wallentin L, Randomized Evaluation of Long-Term Anticoagulation Therapy Investigators. Newly identified events in the RE-LY trial. N Engl J Med 2010;363(19):1875-6.

[41] Patel MR, Mahaffey KW, Garg J, Pan G, Singer DE, Hacke W, et al., ROCKET AF Investigators. Rivaroxaban versus warfarin in nonvalvular atrial fibrillation. N Engl J Med 2011;365(10):88391.

[42] Granger CB, Alexander JH, McMurray JJ, Lopes RD, Hylek EM, Hanna $M$, et al., ARISTOTLE Committees and Investigators. Apixaban versus warfarin in patients with atrial fibrillation. $\mathrm{N}$ Engl J Med 2011;365(11):981-92.

[43] Wann LS, Curtis AB, Ellenbogen KA, Estes NA, 3rd, Ezekowitz MD, Jackman WM, et al., American College of Cardiology Foundation/American Heart Association Task Force. 2011 ACCF/ AHA/HRS focused update on the management of patients with atrial fibrillation (update on dabigatran): A report of the American College of Cardiology Foundation/American Heart Association Task Force on practice guidelines. Circulation 2011;123(10):1144-50.

[44] Connolly SJ, Eikelboom J, Joyner C, Diener HC, Hart R, Golitsyn $S$, et al., AVERROES Steering Committee and Investigators. Apixaban in patients with atrial fibrillation. $N$ Engl $J$ Med 2011;364(9):806-17.

[45] Oldgren J, Alings M, Darius H, Diener HC, Eikelboom J, Ezekowitz MD, et al., RE-LY Investigators. Risks for stroke, bleeding, and death in patients with atrial fibrillation receiving dabigatran or warfarin in relation to the CHADS2 score: a subgroup analysis of the RE-LY trial. Ann Intern Med 2011;155(10):660-7.

[46] Fox KA, Piccini JP, Wojdyla D, Becker RC, Halperin JL, Nessel CC, et al. Prevention of stroke and systemic embolism with rivaroxaban compared with warfarin in patients with non-valvular atrial fibrillation and moderate renal impairment. Eur Heart J 2011;32(19):2387-94.

[47] Wallentin L, Yusuf S, Ezekowitz MD, Alings M, Flather M, Franzosi MG, et al., RE-LY investigators. Efficacy and safety of dabigatran compared with warfarin at different levels of international normalised ratio control for stroke prevention in atrial fibrillation: an analysis of the RE-LY trial. Lancet 2010;376(9745): 975-83.

[48] Stroke prevention in atrial fibrillation with once-daily rivaroxaban: results from ROCKET AF. ESC Congress 2011. Paris, 27-31 August 2011

[49] Garcia D, Libby E, Crowther MA. The new oral anticoagulants. Blood 2010;115(1):15-20.

[50] Ufer M. Comparative efficacy and safety of the novel oral anticoagulants dabigatran, rivaroxaban and apixaban in preclinical and clinical development. Thromb Haemost 2010;103(3): $572-85$.

[51] van Ryn J, Stangier J, Haertter S, Liesenfeld KH, Wienen W, Feuring $M$, et al. Dabigatran etexilate-A novel, reversible, oral direct thrombin inhibitor: interpretation of coagulation assays and reversal of anticoagulant activity. Thromb Haemost 2010; 103(6):1116-27.

[52] Lindhoff-Last E, Samama MM, Ortel TL, Weitz JI, Spiro TE. Assays for measuring rivaroxaban: their suitability and limitations. Ther Drug Monit 2010;32(6):673-9.

[53] Barrett YC, Wang Z, Frost C, Shenker A. Clinical laboratory measurement of direct factor Xa inhibitors: anti-Xa assay is preferable to prothrombin time assay. Thromb Haemost 2010;104(6):1263-71.

[54] Favaloro EJ, Lippi G, Koutts J. Laboratory testing of anticoagulants: the present and the future. Pathology 2011;43(7):68292.

[55] Douketis JD. Dabigatran as anticoagulant therapy for atrial fibrillation. Which patients should receive it, which patients may not need it, and other practical aspects of patient management. Pol Arch Med Wewn 2011;121(3):73-80.

[56] Pengo V, Crippa L, Falanga A, Finazzi G, Marongiu F, Palareti G, et al. Questions and answers on the use of dabigatran and perspectives on the use of other new oral anticoagulants in patients with atrial fibrillation. A consensus document of the Italian Federation of Thrombosis Centers (FCSA). Thromb Haemost 2011;106(5):868-76.

[57] Alexander JH, Becker RC, Bhatt DL, Cools F, Crea F, Dellborg M, et al., APPRAISE Steering Committee and Investigators. Apixaban, an oral, direct, selective factor Xa inhibitor, in combination with antiplatelet therapy after acute coronary syndrome: results of the Apixaban for Prevention of Acute Ischemic and Safety Events (APPRAISE) trial. Circulation 2009;119(22):287785.

[58] Alexander JH, Lopes RD, James S, Kilaru R, He Y, Mohan P, et al., APPRAISE-2 Investigators. Apixaban with antiplatelet therapy after acute coronary syndrome. N Engl J Med 2011;365(8):699708.

[59] Imberti D, Barillari G, Biasioli C, Bianchi M, Contino L, Duce R, et al. Prothrombin complex concentrates for urgent anticoagulation reversal in patients with intracranial haemorrhage. Pathophysiol Haemost Thromb 2008;36(5):259-65.

[60] Masotti L, Pampana A, Pennati P, Landini GC. Esperienza clinica nella gestione dell'emorragia intracranica spontanea in pazienti in terapia con warfarin. Ital J Medicine 2011;5:34-44.

[61] Freeman JV, Zhu RP, Owens DK, Garber AM, Hutton DW, Go AS, et al. Cost-effectiveness of dabigatran compared with warfarin for stroke prevention in atrial fibrillation. Ann Intern Med 2011;154(1):1-11.

[62] Sorensen SV, Kansal AR, Connolly S, Peng S, Linnehan J, BradleyKennedy C, et al. Cost-effectiveness of dabigatran etexilate for the prevention of stroke and systemic embolism in atrial fibril- 
lation: a Canadian payer perspective. Thromb Haemost 2011;105(5):908-19.

[63] Shah SV, Gage BF. Cost-effectiveness of dabigatran for stroke prophylaxis in atrial fibrillation. Circulation 2011;123(22):256270.

[64] Pink J, Lane S, Pirmohamed M, Hughes DA. Dabigatran etexilate versus warfarin in management of non-valvular atrial fibrillation in UK context: quantitative benefit-harm and economic analyses. BMJ 2011;343:d6333.

[65] Masotti L, Di Napoli M, Godoy DA, Rafanelli D, Liumbruno G, Koumpouros $\mathrm{N}$, et al. The practical management of intracerebral hemorrhage associated with oral anticoagulant therapy. Int J Stroke 2011;6(3):228-40.

[66] Ageno W, Garcia D, Aguilar MI, Douketis J, Finazzi G, Imberti D, et al. Prevention and treatment of bleeding complications in patients receiving vitamin $\mathrm{K}$ antagonists. Part 2: Treatment. Am J Hematol 2009;84(9):584-8.

[67] Crowther MA, Warkentin TE. Bleeding risk and the management of bleeding complications in patients undergoing anticoagulant therapy: focus on new anticoagulant agents. Blood 2008; 111(10):4871-9.

[68] Lauer A, Cianchetti FA, van Cott EM, Schlunk F, Schulz E, Pfeilschifter $W$, et al. Anticoagulation with the oral direct thrombin inhibitor dabigatran does not enlarge hematoma volume in experimental intracerebral hemorrhage. Circulation 2011;124(15):1654-62.

[69] Foerch C, Arai K, Jin G, Park KP, Pallast S, van Leyen K, et al. Experimental model of warfarin-associated intracerebral hemorrhage. Stroke 2008;39(12):3397-404.
[70] Levi M. Emergency reversal of antithrombotic treatment. Intern Emerg Med 2009;4(2):137-45.

[71] Morgenstern LB, Hemphill 3rd JC, Anderson C, Becker K, Broderick JP, Connolly Jr ES, et al., American Heart Association Stroke Council and Council on Cardiovascular Nursing. Guidelines for the management of spontaneous intracerebral hemorrhage: a guideline for healthcare professionals from the American Heart Association/American Stroke Association. Stroke 2010;41(9):2108-29.

[72] Eerenberg ES, Kamphuisen PW, Sijpkens MK, Meijers JC, Buller HR, Levi M. Reversal of rivaroxaban and dabigatran by prothrombin complex concentrate: a randomized, placebo-controlled, crossover study in healthy subjects. Circulation 2011;124(14): 1573-9.

[73] Godier A, Miclot A, Le Bonniec B, Durand M, Fischer AM, Emmerich J, et al. Evaluation of prothrombin complex concentrate and recombinant activated factor VII to reverse rivaroxaban in a rabbit model. Anesthesiology 2012;116(1):94-102.

[74] Levi M, Eerenberg E, Kamphuisen PW. Bleeding risk and reversal strategies for old and new anticoagulants and antiplatelet agents. J Thromb Haemost 2011;9(9):1705-12.

[75] Kazmi RS, Lwaleed BA. New anticoagulants: how to deal with treatment failure and bleeding complications. Br J Clin Pharmacol 2011;72(4):593-603.

[76] You JJ, Singer DE, Howard PA et al.; American College of Chest Physicians. Antithrombotic therapy for atrial fibrillation: Antithrombotic Therapy and Prevention of Thrombosis, 9th ed: American College of Chest Physicians Evidence-Based Clinical Practice Guidelines. Chest. 2012;141(2 Suppl):e531S-75S. 